\title{
Review \\ Gender-Related Differences in Chronic Kidney \\ Disease-Associated Vascular Calcification Risk and Potential Risk Mediators: A Scoping Review
}

\author{
Patrick Yihong $\mathrm{Wu}^{1,+}{ }^{1}$, Szu-Ying Lee ${ }^{2,+}$, Ke-Vin Chang ${ }^{3}\left(\mathbb{D}\right.$, Chia-Ter Chao ${ }^{4,5,6, *(\mathbb{D})}$ and Jenq-Wen Huang ${ }^{2,5}$ \\ 1 School of Medicine, National Taiwan University College of Medicine, Taipei 100233, Taiwan; \\ b05401019@ntu.edu.tw \\ 2 Nephrology Division, Department of Internal Medicine, National Taiwan University Hospital Yunlin Branch, \\ Yunlin County 640, Taiwan; isis0525@gmail.com (S.-Y.L.); 007378@ntuh.gov.tw (J.-W.H.) \\ 3 Department of Physical Medicine and Rehabilitation, National Taiwan University Hospital BeiHu Branch, \\ Taipei 10845, Taiwan; kvchang011@gmail.com \\ 4 Graduate Institute of Toxicology, National Taiwan University College of Medicine, Taipei 100233, Taiwan \\ 5 Nephrology Division, Department of Internal Medicine, National Taiwan University College of Medicine, \\ Taipei 100233, Taiwan \\ 6 Nephrology Division, Department of Internal Medicine, National Taiwan University Hospital BeiHu Branch, \\ Taipei 10845, Taiwan \\ * Correspondence: b88401084@gmail.com; Tel.: +886-2-23717101 (ext. 6531); Fax: +886-2-23717101 \\ + Contribute equally.
}

check for

updates

Citation: Wu, P.Y.; Lee, S.-Y.; Chang, K.-V.; Chao, C.-T.; Huang, J.-W. Gender-Related Differences in Chronic Kidney Disease-Associated Vascular Calcification Risk and Potential Risk Mediators: A Scoping Review. Healthcare 2021, 9, 979. https://doi.org/10.3390/

healthcare 9080979

Academic Editor: Jang-Hee Cho

Received: 21 June 2021

Accepted: 28 July 2021

Published: 1 August 2021

Publisher's Note: MDPI stays neutral with regard to jurisdictional claims in published maps and institutional affiliations.

Copyright: (c) 2021 by the authors. Licensee MDPI, Basel, Switzerland. This article is an open access article distributed under the terms and conditions of the Creative Commons Attribution (CC BY) license (https:// creativecommons.org/licenses/by/ $4.0 /)$.

\begin{abstract}
Vascular calcification (VC) involves the deposition of calcium apatite in vascular intima or media. Individuals of advanced age, having diabetes mellitus or chronic kidney disease (CKD) are particularly at risk. The pathogenesis of CKD-associated VC evolves considerably. The core driver is the phenotypic change involving vascular wall constituent cells toward manifestations similar to that undergone by osteoblasts. Gender-related differences are observed regarding the expressions of osteogenesis-regulating effectors, and presumably the prevalence/risk of CKD-associated VC exhibits gender-related differences as well. Despite the wealth of data focusing on gender-related differences in the risk of atherosclerosis, few report whether gender modifies the risk of VC, especially CKD-associated cases. We systematically identified studies of CKD-associated VC or its regulators/modifiers reporting data about gender distributions, and extracted results from 167 articles. A significantly higher risk of CKD-associated VC was observed in males among the majority of original investigations. However, substantial heterogeneity exists, since multiple large-scale studies yielded neutral findings. Differences in gender-related VC risk may result from variations in VC assessment methods, the anatomical segments of interest, study sample size, and even the ethnic origins of participants. From a biological perspective, plausible mediators of gender-related VC differences include body composition discrepancies, alterations involving lipid profiles, inflammatory severity, diversities in matrix Gla protein (MGP), soluble Klotho, vitamin D, sclerostin, parathyroid hormone (PTH), fibroblast growth factor-23 (FGF-23), and osteoprotegerin levels. Based on our findings, it may be inappropriate to monotonously assume that male patients with CKD are at risk of VC compared to females, and we should consider more background in context before result interpretation.
\end{abstract}

Keywords: aortic calcification; chronic kidney disease; dialysis; fetuin-A; gender; end-stage renal disease; fibroblast growth factor-23; Klotho; mineral bone disorder; osteoprotegerin; sclerostin; sex; vascular calcification; valvular calcification; vitamin D

\section{Vascular Calcification (VC): An Introduction}

The ectopic deposition of calcium-containing crystals in vascular intima or media is termed VC and involves large conduit arteries and small ones as well. VC frequently affects individuals of advanced age and those with accelerated biological ageing related to 
diabetes mellitus (DM) or chronic kidney disease (CKD). Findings from large populationbased cohorts identified a VC prevalence of $40 \%$ to $60 \%$ and $30 \%$ to $40 \%$ among older [1,2] and middle-aged adults [3]. Meta-analyses revealed that the VC prevalence rose to $60 \%$ among patients with CKD regardless of age [4] and also increased in those with DM [5]. Calcifications affecting conduit arteries lead to vessel stiffening, increased peripheral resistance and the risk of hypertension, cardiac hypertrophy, heart failure and cardiovascular mortality. Calcifications involving microcirculation aggravate tissue perfusion through microvascular dysfunction and they induce silent ischemic episodes [6], leading to a higher risk of cardiovascular events [7,8]. VC-related adverse outcome influences become more prominent among those with CKD [9]. Valvular calcification portends a similarly worse outcome among this population [10]. VC further elevates their risk of having rapid renal function decline [11], developing morbidities including malignancies, hip fractures, pneumonia, and detrimental phenotypes like frailty $[12,13]$. The understanding of pathogenesis related to CKD-associated VC evolves considerably but remains elusive. It is now believed that vascular smooth muscle cells (VSMCs) and/or their neighboring constituents adopt an osteoblast-like phenotype and these trans-differentiated cells secrete osteoid matrices under pervasive exposure, leading to VC [14]. This calcification process is further augmented by redox imbalance, epigenetic changes, uremic milieu, the reduction in endogenous anticalcific defense machineries, extracellular vesicle secretion and the dysregulated microRNA network [15-18].

\section{Risk Factors of CKD-Associated VC: Gender Matters?}

$\mathrm{VC}$ is one of the CKD-mineral bone disorder (CKD-MBD) components, which encompass divalent ion dysregulation (dyscalcemia, hyperphosphatemia), osteodystrophy, and ectopic calcification involving soft tissues. There are two types of $\mathrm{VC}$, intimal and medial calcification. The former resides within atherosclerotic plaques based on tunica intima, and shares atherosclerotic risk factors including an advanced age, genetic susceptibility such as polymorphisms involving ApoE and Lp(a), obesity, DM and/or insulin resistance, other cardiovascular morbidities, etc. The latter type is associated with atypical risk factors such as uremic toxin exposure, osteoporosis/sarcopenia, chronic inflammation, and elevated oxidative stress/glycation endproducts [19]. Medial calcification is proposed to be more characteristic of CKD-associated VC relative to intimal calcification, although the incidence of both disease categories rises in the CKD population.

Traditional belief maintains that males have a significantly higher prevalence of atherosclerosis, are more likely to have accelerated lesion progression, be symptomatic, and to have more severe/unstable plaque features compared to females [20]. There are also studies suggesting that the influence of beneficial lifestyle changes on the risk of atherosclerosis may have gender-related differences [21]. On the contrary, females tend to occupy a greater proportion of patients with atherosclerosis or ischemic heart disease than males after 75 years [22]. Gender-related differences in atherosclerosis are mainly attributed to estrogenic action, since younger females with primary ovarian insufficiency have a significantly elevated risk of ischemic heart events and overall mortality than those without [23]. Despite the wealth of data regarding gender differences in atherosclerotic risk, few address whether gender modifies VC risk, especially CKD-associated VC. A recent study showed that males had a 3-fold higher risk of coronary artery calcification (CAC) compared to females, and the former had a more diffuse distribution of calcification than the latter [24]. Consequently, we performed a scoping literature review aiming to summarize the existing knowledge regarding gender-related differences in VC prevalence, severity, risk, and potential mediators.

\section{Literature Search Strategy}

We adopted a systematic approach to identify articles focusing on VC in patients across all stages of CKD, using the following MeSH or Emtree keywords: 'vascular calcification', 'male' or 'female', and 'renal insufficiency, chronic' or 'renal replacement therapy', from 
databases including PubMed, MEDLINE, EMBASE, Google Scholar, and Cochrane Reports between 1968 and 6 May 2021. Inclusion criteria were original investigations involving human subjects, reporting data of the gender variable and any types of VC, pulse wave velocity $(\mathrm{PWV})$, serum calcification propensity $\left(\mathrm{T}_{50}\right)$, or potential functional regulators of $\mathrm{VC}$, among populations with various stages of CKD. Identified studies were initially screened by two investigators (P.Y.W. and C.T.C.) independently. This was followed by the application of exclusion criteria, consisting of review articles, those without an abstract available for screening, those that did not provide data on gender-related differences in the prevalence/course/risk of VC or its related regulators, or those that focused on non-CKD patients. We subsequently reviewed the abstract of identified articles and their reference lists to uncover additional studies containing original data of similar topics. Discrepancies between the two reviewers regarding article eligibility were resolved by arbitration from a senior investigator (J.W.H.).

We extracted relevant parameters from the identified studies, including the year and authors of publication, the stages of participants' CKD at baseline, methods for measuring VC status and severities, the distribution of gender and/or the gender as a predictor of VC or its regulators, and results from univariate and multivariate analyses of clinical features between males and females. We organized results according to the following categories: gender-related difference in the prevalence, severities and/or courses of VC; gender as a risk factor for incident/worsening $\mathrm{VC}$; and gender as a modifier for $\mathrm{VC}$ risk.

\section{Summary of Findings}

A total of 893 studies were screened initially (Figure 1). After exclusion of articles that met our exclusion criteria following the title/abstract/full-text review, 167 articles were retained for summarization. In most studies of non-dialysis CKD patients, the estimated glomerular filtration rate (eGFR) was obtained based on the Modification of Diet in Renal Disease (MDRD) formula, while some earlier studies used elevated serum creatinine to identify those with CKD. Staging of CKD, when available, was done according to the Kidney Disease Improving Global Outcome (KDIGO) classification [25]. For the estimation of $\mathrm{VC}$ risk in multivariate analyses, factors that were adjusted for included at least age and gender and could further include interfering parameters such as body size, comorbidities, medications, and/or laboratory indicators.

\subsection{Gender-Related Differences in the Prevalence and/or Severity of CKD-Associated VC}

In total, $101(60.5 \%)$ original investigations evaluated the differences in the prevalence and / or severity of CKD-associated VC between different genders [26-126]. Among these articles, $27(26.7 \%), 64(63.4 \%)$ and $10(9.9 \%)$ included patients with non-dialysis CKD, dialysis-dependent CKD, and renal transplant recipients, respectively (Supplementary Table S1). In those involving patients with non-dialysis CKD, one-third $(\mathrm{n}=9)$ found no differences in VC prevalence/risk between genders, while two-thirds $(n=18)$ reported that males had a higher VC prevalence/severity than females. Interestingly, in reports involving patients with non-dialysis CKD, those directly assessing VC based on coronary computed tomography (CT) or plain radiography for aortas tended to derive results that suggested higher male VC prevalence/severity, when the case number was large. On the other hand, studies that involved the assessment of PWV or $\mathrm{T}_{50}$ tended to have neutral results (Supplementary Table S1).

In studies involving patients with dialysis-dependent CKD, $37(57.8 \%)$ found no differences in VC prevalence/severity between genders, while $22(34.4 \%)$ found that males had a higher VC prevalence than females (Supplementary Table S1). On the contrary, 5 $(7.8 \%)$ reports identified that females had a higher VC prevalence than males. If coronary $\mathrm{VC}$ was the phenotype of interest in studies involving patients with dialysis-dependent CKD, males were more likely to have a higher prevalence/severity of VC than females (neutral vs. male higher, 5 out of $37(13.5 \%)$ vs. 8 out of 22 (36.4\%) studies using Agatston scores as surrogates). Reports that identified a higher VC prevalence/severity in dialysis- 
dependent CKD females than males tended to originate from Asian countries and involved carotid artery calcification (Supplementary Table S1).

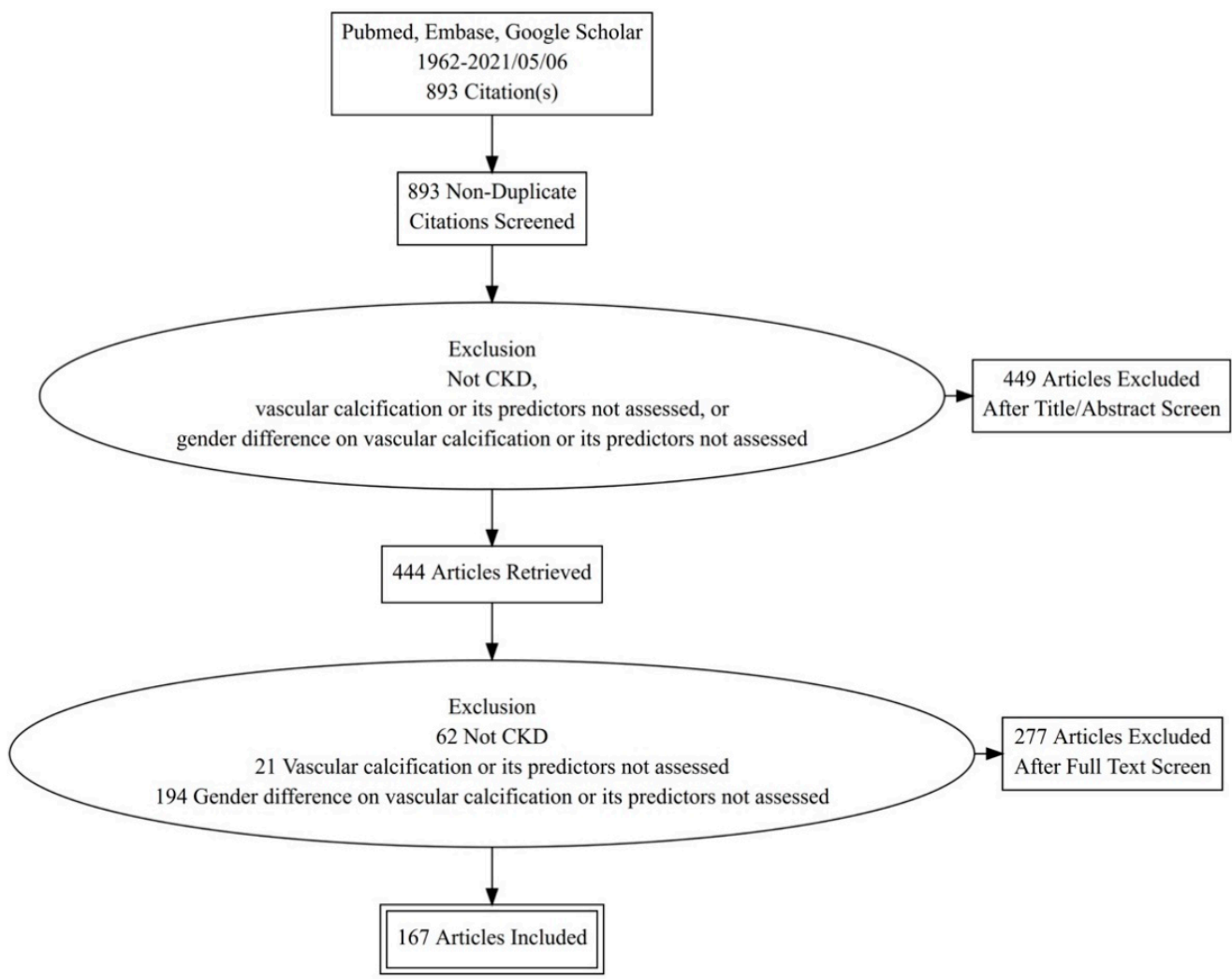

Figure 1. Literature selection algorithm of this review. CKD, chronic kidney disease.

In studies involving patients receiving renal transplant (CKD stage 5T), $6(60 \%)$ found no differences in VC prevalence/severity between genders, while $4(40 \%)$ reported more males with a higher VC prevalence/severity than females (Supplementary Table S1). Similar to studies involving dialysis-dependent CKD patients, if coronary VC was the phenotype of interest, males were more likely to be reported as having higher prevalence/severity of VC than females (neutral vs. male higher, 1 out of $6(16.7 \%)$ vs. 2 out of $4(50 \%)$ studies using Agatston scores as surrogates).

Few studies adopted a histopathological approach to identify gender-related differences in CKD-associated VC prevalence/severity $(n=5 ; 5.0 \%)$, and even fewer addressed gender-related differences in valvular calcification $(\mathrm{n}=3,3.0 \%)$ (Supplementary Table S1). There were reports suggesting neutral results or higher male prevalence/severity of VC using the histopathological approach, while none of the reports addressing valvular calcification identified gender-related differences in prevalence.

\subsection{Gender-Related Differences in the Adjusted Risk of CKD-Associated VC Presence or Severity}

A total of $62(37.1 \%)$ original investigations reported gender-associated adjusted risk of having higher prevalence/severity of VC among patients with CKD [40,42,44,47,48,58,63-65,67,68,84,86,88,90,93,95,100-102,106,112,113,116,117,119-155]. Among these articles, 17 (27.4\%), 40 (64.5\%) and 5 (8.1\%) included patients with nondialysis $\mathrm{CKD}$, dialysis-dependent $\mathrm{CKD}$, and renal transplant recipients, respectively (Supplementary Table S2). In those involving patients with non-dialysis CKD, only 3 (17.6\%) found no differences in VC risk between genders, while 14 (82.4\%) reported that males had a higher adjusted risk of VC than females. A forest plot illustrating the risk of VC presence or greater severity associated with male gender in those with non-dialysis CKD is shown in Figure 2. Among studies enrolling more than 100 patients, most concluded 
that males exhibited a significantly higher risk of having VC involving coronary arteries, aortas or higher PWVs than females (Supplementary Table S2). The male-to-female odds ratio (ORs) of developing VC ranged between 4.2 and 43, depending on the cohort size and the combinations of adjusted variables, regardless of assessment methods for VC. In those involving patients with dialysis-dependent CKD, relatively few $(n=13 ; 32.5 \%)$ found no gender-related alterations in VC risk, and reports identifying a higher VC risk among males $(\mathrm{n}=25 ; 62.5 \%)$ significantly outnumbered those identifying a higher VC risk among females $(\mathrm{n}=2 ; 5 \%)$ (Supplementary Table S2). A forest plot illustrating the risk of VC presence or greater severity associated with male gender in those with dialysisdependent CKD is shown in Figure 3. The male-to-female ORs or relative risks (RRs) of CKD-associated VC were between 1.9 and 10.5. It appears that the male-to-female OR/RRs for VC tend to be lower if aortic calcification was considered (between 2.2 and 3.3) and higher if CAC was studied (between 2.8 and 10.5) (Supplementary Table S2). Reports identifying a higher CKD-associated VC risk among females than males focused on aortic calcification and carotid artery calcification. Among studies involving patients receiving renal transplantation, most $(n=4 ; 80 \%)$ concluded that males had a significantly higher risk of VC than females, and 75\% of them assess CAC (Supplementary Table S2).

\begin{tabular}{lrrr} 
Study name & \multicolumn{3}{c}{ Statistics for each study } \\
& $\begin{array}{r}\text { Odds } \\
\text { ratio }\end{array}$ & $\begin{array}{c}\text { Lower } \\
\text { limit }\end{array}$ & $\begin{array}{c}\text { Upper } \\
\text { limit }\end{array}$ \\
Vipattawat et al. 2014 (1) & 2.490 & 0.869 & 7.133 \\
Vipattawat et al. 2014 (2) & 2.020 & 0.708 & 5.763 \\
Porter et al. 2007 & 43.713 & 3.305 & 578.159 \\
Stavroulopoulos et al. 2011 & 27.808 & 1.625 & 475.919 \\
Harada et al. 2014 & 4.920 & 2.069 & 11.697 \\
Morena et al. 2009 & 4.950 & 2.361 & 10.376 \\
Sigrist et al. 2007 & 8.820 & 1.822 & 42.697 \\
Golembiewska et al. 2020 & 4.400 & 1.671 & 11.589 \\
Dai et al. 2020 & 6.670 & 2.530 & 17.582 \\
Craver et al. 2013 & 4.218 & 1.319 & 13.485
\end{tabular}

\section{Odds ratio and $95 \% \mathrm{Cl}$}
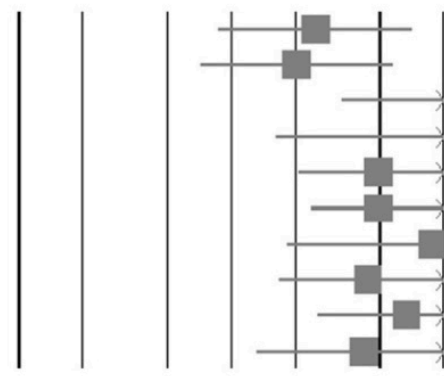

$\begin{array}{lllllll}0.1 & 0.2 & 0.5 & 1 & 2 & 5 & 10\end{array}$

Figure 2. A forest plot showing the male to female risk among patients with non-dialysis chronic kidney disease.

\begin{tabular}{lrrr} 
Study name & \multicolumn{3}{c}{ Statistics for each study } \\
\cline { 2 - 4 } & $\begin{array}{r}\text { Odds } \\
\text { ratio }\end{array}$ & $\begin{array}{c}\text { Lower } \\
\text { limit }\end{array}$ & $\begin{array}{c}\text { Upper } \\
\text { limit }\end{array}$ \\
Cai et al. 2015 & 0.549 & 0.113 & 2.664 \\
Coll et al. 2011 & 1.570 & 0.692 & 3.561 \\
Fusaro et al. 2015 & 1.520 & 0.892 & 2.591 \\
Moldovan et al. 2011 & 7.226 & 1.138 & 45.883 \\
Mazzaferro et al. 2007 & 10.500 & 3.202 & 34.427 \\
Adragao et al. 2004 & 7.470 & 2.911 & 19.171 \\
Okamoto et al. 2018 & 3.290 & 1.269 & 8.526 \\
Turan et al. 2013 & 2.790 & 1.301 & 5.984 \\
Schlieper et al. 2009 & 2.320 & 1.190 & 4.522 \\
Schlieper et al. 2008 & 5.080 & 2.178 & 11.849 \\
Turan et al. 2016 & 4.140 & 2.012 & 8.519 \\
Nishizawa et al. 2005 & 3.380 & 1.289 & 8.861 \\
Ishimura et al. 2002 & 3.380 & 1.289 & 8.861 \\
Ishimura et al. 2004 & 2.339 & 1.466 & 3.732
\end{tabular}

\section{Odds ratio and $95 \% \mathrm{Cl}$}

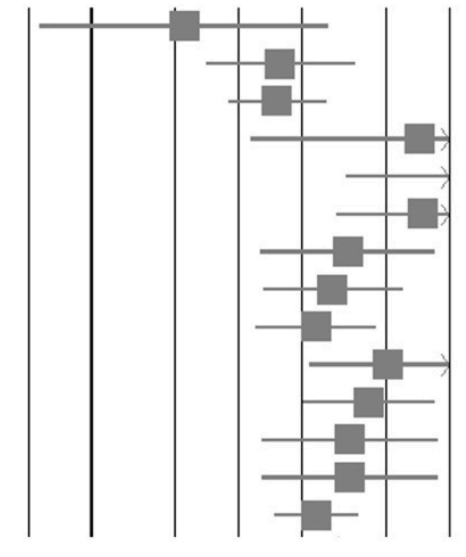

$\begin{array}{lllllll}0.1 & 0.2 & 0.5 & 1 & 2 & 5 & 10\end{array}$

Figure 3. A forest plot showing the male to female risk among patients with dialysis-dependent chronic kidney disease. 


\subsection{Potential Mediators of Gender-Related Differences in CKD-Associated VC Risk}

A total of 53 original investigations addressed potential modifiers of the genderVC relationship among patients with CKD, based on our literature summary $[36,40,43,58,59,61,66,86,106,107,110,115,129,130,134,152,156-192]$. We divided these mediators into 7 categories: genetic susceptibility, body composition and nutrition/inflammation status, medication, epigenetic mediators, divalent ion/electrolyte balances, osteogenesis regulators, and miscellaneous (Table 1). Studies involving polymorphisms of the vitamin K metabolism-related gene (VKORC1) and calcification inhibitors (matrix Gla protein; MGP) did not find gender-related differences in allelic distributions. In terms of body composition, males had significantly higher pericardial fat areas, low density lipoprotein (LDL) cholesterol, and angiotensin-converting enzyme 2 (ACE2) levels but lower total fat mass, adiponectin, and high density lipoprotein (HDL) cholesterol than females (Table 1). Fluid status, either in the form of extracellular fluid volume or serum copeptin levels, did not exhibit gender-related differences when CKD-associated $\mathrm{VC}$ risk was considered. There were studies addressing gender-related differences in CKDassociated VC risk modifying medication (statin) or microRNA levels, but the results were all neutral. Divalent ions and electrolytes such as serum phosphorus, calcium, and magnesium were reported to influence the risk of CKD-associated VC [193]; in our literature summary, females were found to have a higher probability of hyperphosphatemia than males (Table 1), but there was no difference in serum magnesium or calciuria levels. Thirty $(56.6 \%)$ reports intended to examine gender-related differences in multiple osteogenesis regulator levels, including MGP $(\mathrm{n}=2 ; 6.7 \%)$, fetuin-A $(\mathrm{n}=4 ; 13.3 \%)$, fibroblast growth factor-23 (FGF-23) $(\mathrm{n}=2 ; 6.7 \%)$, osteoprotegerin $(\mathrm{n}=8 ; 26.7 \%)$, parathyroid hormone $(\mathrm{PTH})(\mathrm{n}=2 ; 6.7 \%)$, sclerostin $(\mathrm{n}=8 ; 26.7 \%)$, soluble Klotho $(\mathrm{n}=3 ; 10 \%)$, and vitamin $\mathrm{D}$ $(\mathrm{n}=2 ; 6.7 \%)$. No gender-related differences were observed regarding fetuin-A, while most studies concluded that males had significantly lower dephosphorylated uncarboxylated MGP and Klotho but higher sclerostin and vitamin D levels than females (Table 1). Studies focusing on gender-related differences in PTH, FGF-23, and osteoprotegerin levels among patients with CKD had controversial findings; those addressing PTH or FGF-23 had either neutral results or higher levels in females than males. Studies addressing osteoprotegerin yielded neutral, male higher, or female higher results. Interestingly, reports garnering higher patient numbers uniformly concluded that females had higher osteoprotegerin levels than males (Table 1). Finally, several studies focusing on VC identified gender-related differences in signal peptide-CUB-EGF domain-containing protein 1 (SCUBE1) and tissue advanced glycation (Table 1), which might bear potential influences on gender-related VC risk.

Table 1. Potential gender-related modifiers of vascular calcification in existing studies.

\begin{tabular}{|c|c|c|c|c|c|c|c|c|}
\hline Authors & Country & Time & $\begin{array}{l}\text { CKD } \\
\text { Stages }\end{array}$ & $\begin{array}{l}\text { Sample } \\
\text { Size }\end{array}$ & $\begin{array}{c}\text { Gender } \\
\text { Effect }\end{array}$ & Data & Potential Modifiers & Ref. \\
\hline \multicolumn{9}{|c|}{ Genetic susceptibility } \\
\hline Holden et al. & Canada & 2014 & $3-5$ & 167 & Neutral & CC vs. CG/GG, male $68 \%$ vs. $55 \%, p=0.11$ & $\begin{array}{c}\text { VKORC1 (vitamin K } \\
\text { epoxide reductase } \\
\text { complex 1) } \\
\text { polymorphism }\end{array}$ & [166] \\
\hline $\begin{array}{l}\text { Yoshikawa } \\
\text { et al. }\end{array}$ & Japan & 2013 & 5D (HD) & 134 & Neutral & $\begin{array}{c}\text { CC vs. CT vs. TT genotype, male } 14 \% \text { vs. } \\
48 \% \text { vs. } 33 \%, p=0.53\end{array}$ & $\begin{array}{l}\text { MGP Genotype } \\
\text { T-138C }\end{array}$ & [110] \\
\hline \multicolumn{9}{|c|}{ Body composition and inflammatory status } \\
\hline Axelsson et al. & Sweden & 2008 & 5 & 198 & Male lower & Male $\beta=-1.68, p<0.001$ & Total fat mass & [157] \\
\hline Park et al. & Korean & 2018 & $1-5$ & 1741 & Neutral & $\begin{array}{c}\text { Quartile } 1 \text { (lowest) vs. } 2 \text { vs. } 3 \text { vs. } 4 \text {, male } \\
80.6 \% \text { vs. } 59.0 \% \text { vs. } 39.7 \% \text { vs. } 39.6, p<0.001 \\
\text { Male } \beta=0.095(-0.421 \sim 0.231), p=0.566\end{array}$ & ECF excess & [179] \\
\hline
\end{tabular}


Table 1. Cont.

\begin{tabular}{|c|c|c|c|c|c|c|c|c|}
\hline Authors & Country & Time & $\begin{array}{l}\text { CKD } \\
\text { Stages }\end{array}$ & $\begin{array}{l}\text { Sample } \\
\text { Size }\end{array}$ & $\begin{array}{l}\text { Gender } \\
\text { Effect }\end{array}$ & Data & Potential Modifiers & Ref. \\
\hline Choi et al. & Korea & 2019 & 5D (HD) & 97 & Neutral & $\begin{array}{l}\text { Marker count } 2 \text { vs. } 1 \text { vs. } 0 \text {, male } 61.5 \% \text { vs. } \\
41.9 \% \text { vs. } 34.1 \%, p=0.216\end{array}$ & $\begin{array}{l}\text { Nutrition (albumin) } \\
\text { and inflammation } \\
\text { (CRP) markers }\end{array}$ & [43] \\
\hline $\begin{array}{l}\text { Golembiewska } \\
\text { et al. }\end{array}$ & Sweden & 2020 & 5 & 149 & Neutral & $\begin{array}{l}\text { High vs. middle vs. low levels, male } 71 \% \text { vs. } \\
67 \% \text { vs. } 65 \%, p=0.84 \\
\text { Regression model, male } \beta-0.08, p=0.31\end{array}$ & Copeptin & [134] \\
\hline Harada et al. & Brazil & 2014 & $2-5$ & 117 & $\begin{array}{l}\text { Male more } \\
\text { severe }\end{array}$ & $\begin{array}{l}\text { Area in males vs. females, } 220.4 \pm 110.5 \text { vs. } \\
\qquad 135.4 \pm 76.0, p<0.001\end{array}$ & Pericardial fat & [58] \\
\hline Turan et al. & Turkey & 2013 & 5D (HD) & 191 & Neutral & $\begin{array}{l}\text { Low vs. middle vs. high, female } 46 \% \text { vs. } 39 \% \\
\text { vs. } 58 \%, p=0.1\end{array}$ & Epicardial fat & [152] \\
\hline Miyatake et al. & Japan & 2020 & $5 \mathrm{~T}$ & 50 & $\begin{array}{l}\text { Female } \\
\text { higher HDL, } \\
\text { adiponectin } \\
\text { but lower } \\
\text { LDL }\end{array}$ & $\begin{array}{c}\text { For LDL-C, male vs. female } 113.0(97.0-132.5) \\
\text { vs. } 90.0 \text { (76.5-98.3), } p<0.01 \\
\text { For HDL-C, male vs. female } 57.0(51.0-67.0) \\
\text { vs. } 78.0(66.8-96.5), p<0.01 \\
\text { For HMW-ADPN, male vs. female } 2.48 \\
\text { (1.62-3.33) vs. } 4.52(3.02-6.79), p<0.01 \\
\text { For LMW-ADPN, male vs. female } 1.67 \\
\text { (1.14-1.89) vs. } 2.26(1.85-2.83), p<0.01 \\
\text { For CTRP9, male vs. female } 2.08(2.01-2.13) \\
\text { vs. } 2.03(2.00-2.07), p>0.05\end{array}$ & $\begin{array}{c}\text { Lipid profile, } \\
\text { circulating } \\
\text { C1q/TNF-a related } \\
\text { protein-9 (CTRP9), } \\
\text { and adiponectin }\end{array}$ & [86] \\
\hline Zhang et al. & China & 2015 & 5D (HD) & 90 & Male higher & $\begin{array}{c}\leq 30 \text { vs. } 30-60 \text { vs. } \geq 60 \mathrm{U} / \mathrm{L} \text {, male } 29.4 \% \text { vs. } \\
46.1 \% \text { vs. } 85.7 \%, p<0.001 \\
\text { Male correlation coefficient } 0.362, p<0.001\end{array}$ & Serum ACE2 & [190] \\
\hline \multicolumn{9}{|l|}{ Medications } \\
\hline Chen et al. & Sweden & 2017 & $5 \mathrm{D}, 5 \mathrm{~T}$ & 240 & Neutral & $\begin{array}{l}\text { Users vs. non-users, male } 65 \% \text { vs. } 62 \% \text {, } \\
\qquad p=0.678\end{array}$ & Statin use & [41] \\
\hline \multicolumn{9}{|c|}{ Epigenetic mediators } \\
\hline Chao et al. & Taiwan & 2019 & $5 \mathrm{D}$ & 223 & Neutral & $\begin{array}{l}\text { High vs. low levels, female } 63 \% \text { vs. } 51 \% \text {, } \\
\qquad p=0.14\end{array}$ & microRNA-125b & [160] \\
\hline \multicolumn{9}{|c|}{ Divalent ion/electrolyte balance } \\
\hline Block et al. & $\begin{array}{l}\text { United } \\
\text { States }\end{array}$ & 1998 & $5 \mathrm{D}(\mathrm{HD})$ & 6407 & $\begin{array}{l}\text { Female } \\
\text { higher }\end{array}$ & Male OR $0.774, p=0.0001$ & $\begin{array}{l}\text { Serum phosphorus }> \\
6.5 \mathrm{mg} / \mathrm{dL}\end{array}$ & [158] \\
\hline Zou et al. & China & 2016 & $1-5$ & 296 & Neutral & $\begin{array}{l}\text { Tertile } 1 \text { (lowest) vs. } 2 \text { vs. } 3 \text {, male } 60.2 \% \text { vs. } \\
50.0 \% \text { vs. } 61.2 \%, p=0.21\end{array}$ & Serum phosphorus & [192] \\
\hline $\begin{array}{l}\text { Karsli } \\
\text { Ceppioğlu } \\
\text { et al. }\end{array}$ & Turkey & 2011 & $3-5$ & 84 & $\begin{array}{l}\text { Female } \\
\text { higher }\end{array}$ & $\begin{array}{l}\text { Female vs. male, higher in the former, } \\
\qquad p=0.002\end{array}$ & Serum copper & [170] \\
\hline Ikee et al. & Japan & 2016 & $5 \mathrm{D}(\mathrm{HD})$ & 86 & Neutral & $\begin{array}{c}\text { Male vs. female, } 2.51 \pm 0.38 \text { vs. } 2.42 \pm 0.33 \\
\qquad p=0.52\end{array}$ & Serum magnesium & [167] \\
\hline Stolic et al. & Serbia & 2016 & 5D (HD) & 88 & Neutral & $\begin{array}{l}\text { Male vs. female, mean } 1.2(0.7-1.6) \text { vs. } 1.2 \\
\quad(0.8-1.5) \mathrm{mmol} / \mathrm{L}, p=0.896\end{array}$ & Serum magnesium & [185] \\
\hline Ramalho et al. & Brazil & 2019 & $3-4$ & 356 & Neutral & $\begin{array}{c}\text { Tertile } 1 \text { (lowest) vs. } 2 \text { vs. } 3,59 \% \text { vs. } 64 \% \text { vs. } \\
66 \%, p=0.5 \\
\text { Male } \beta=0.22(-0.02 \sim 0.45), p=0.07\end{array}$ & $\begin{array}{l}\text { Urinary calcium } \\
\text { excretion }\end{array}$ & [180] \\
\hline \multicolumn{9}{|c|}{ Osteogenesis/mineralization regulators } \\
\hline Aoun et al. & Lebanon & 2017 & 5D (HD) & 50 & Male lower & $\begin{array}{c}<5000 \text { vs. }>5000 \mathrm{pmol} / \mathrm{L} \text {, male } 68.3 \% \text { vs. } \\
22.2 \%, p=0.02\end{array}$ & Dp-ucMGP & [156] \\
\hline Schlieper et al. & Serbia & 2011 & 5 & 188 & Neutral & $\begin{array}{c}<6139 \text { vs. }>6139 \mathrm{pmol} / \mathrm{L} \text {, female OR } 0.62 \\
(0.35-1.11), p=0.11\end{array}$ & dp-cMGP & [182] \\
\hline Okamoto et al. & Japan & 2018 & 5D (HD) & 230 & Neutral & $\begin{array}{c}<0.213 \text { vs. } \geq 0.213 \mathrm{~g} / \mathrm{L}, \text { male } 61 \% \text { vs. } 68 \% \\
p=0.400 \\
\text { Male OR } 0.48(0.22-1.02), p=0.056\end{array}$ & Fetuin-A & [178] \\
\hline Chen et al. & Taiwan & 2013 & 5D (HD) & 238 & Neutral & $\begin{array}{c}\text { Tertile } 1 \text { (lowest) vs. } 2 \text { vs. } 3 \text {, female } 55 \% \text { vs. } \\
48 \% \text { vs. } 57 \%, p=0.5\end{array}$ & Fetuin-A & [161] \\
\hline $\begin{array}{l}\text { Stenvinkel } \\
\text { et al. }\end{array}$ & Sweden & 2005 & 5 & 258 & Neutral & $\begin{array}{l}\text { Male vs. female, median } 0.225 \text { vs. } 0.223 \mathrm{~g} / \mathrm{L} \text {, } \\
\qquad p>0.05\end{array}$ & Fetuin-A & [184] \\
\hline
\end{tabular}


Table 1. Cont.

\begin{tabular}{|c|c|c|c|c|c|c|c|c|}
\hline Authors & Country & Time & $\begin{array}{l}\text { CKD } \\
\text { Stages }\end{array}$ & $\begin{array}{l}\text { Sample } \\
\text { Size }\end{array}$ & $\begin{array}{l}\text { Gender } \\
\text { Effect }\end{array}$ & Data & Potential Modifiers & Ref. \\
\hline Metry et al. & Sweden & 2008 & 5D (HD) & 222 & Neutral & $\begin{array}{l}\text { High Fetuin-A/Low CRP vs. High } \\
\text { Fetuin-A/High CRP vs. Low Fetuin-A/Low } \\
\text { CRP vs. Low Fetuin-A/High CRP, } 60.9 \% \text { vs. } \\
52.5 \% \text { vs. } 44.4 \% \text { vs. } 58.8 \%, p>0.05\end{array}$ & Fetuin-A and CRP & [174] \\
\hline Turan et al. & Turkey & 2016 & 5D (HD) & 224 & Neutral & $\begin{array}{c}<35.5 \text { vs. } 35.5-123.4 \text { vs. }>123.4 \mathrm{pg} / \mathrm{mL} \text {, male } \\
56.6 \% \text { vs. } 46.5 \% \text { vs. } 50.0 \%, p=0.38\end{array}$ & FGF-23 & [106] \\
\hline Nishiura et al. & Japan & 2009 & 5D (HD) & 99 & Male higher & $\begin{array}{l}\text { Low vs. high, male } 56 \% \text { vs. } 75.5 \%, p=0.057 \\
\text { Male HR } 3.034(1.028-8.948), p=0.044\end{array}$ & Osteoprotegerin & [177] \\
\hline Hou et al. & Taiwan & 2019 & 5D (HD) & 120 & $\begin{array}{l}\text { Female } \\
\text { lower }\end{array}$ & $\begin{array}{c}\text { 72.97-237.13 vs. } 237.36-323.54 \text { vs. } \\
\text { 329.61-835.78 pg/L, female } 62.5 \% \text { vs. } 55.0 \% \\
\text { vs. } 32.5 \%, p=0.008\end{array}$ & Osteoprotegerin & [61] \\
\hline Sigrist et al. & $\begin{array}{l}\text { United } \\
\text { Kingdom }\end{array}$ & 2009 & $3-4$ & 134 & Neutral & $\begin{array}{c}\leq 25 \text { vs. }>25 \mathrm{pmol} / \mathrm{L}, \text { male } 63 \% \text { vs. } 70 \%, p= \\
0.47\end{array}$ & Osteoprotegerin & [183] \\
\hline $\begin{array}{l}\text { Nakashima } \\
\text { et al. }\end{array}$ & Japan & 2011 & 5D (HD) & 151 & Neutral & Male $\beta=0.531, p=0.51$ & Osteoprotegerin & [175] \\
\hline Scialla et al. & $\begin{array}{l}\text { United } \\
\text { States }\end{array}$ & 2011 & $1-5$ & 351 & $\begin{array}{l}\text { Female } \\
\text { higher }\end{array}$ & $\begin{array}{c}1.21-5.03 \text { vs. } 5.05-7.45 \text { vs. } 7.46-22.31 \mathrm{pmol} / \mathrm{L} \\
\text { female } 24.8 \% \text { vs. } 26.5 \% \text { vs. } 40.2 \%, p=0.02 \\
\text { Female related OPG } \% \text { difference } 10.2 \%, p= \\
0.05\end{array}$ & Osteoprotegerin & [181] \\
\hline Gupta et al. & Hungary & 2021 & 5D (HD) & 982 & $\begin{array}{l}\text { Female } \\
\text { higher }\end{array}$ & $\begin{array}{c}<3.2 \text { vs. } 3.2-4.39 \text { vs. }>4.39 \mathrm{pmol} / \mathrm{L} \text {, male } 63 \% \\
\text { vs. } 57 \% \text { vs. } 52 \%, p=0.015\end{array}$ & Osteoprotegerin & [165] \\
\hline Nemeth et al. & Hungary & 2015 & $5 \mathrm{~T}$ & 993 & Male lower & $\begin{array}{c}<3.2 \text { vs. } 3.2-4.39 \text { vs. }>4.39 \mathrm{pmol} / \mathrm{L} \text {, male } 63 \% \\
\text { vs. } 58 \% \text { vs. } 52 \%, p=0.02\end{array}$ & Osteoprotegerin & [176] \\
\hline Chae et al. & Korea & 2018 & $1-5$ & 1832 & Neutral & $\begin{array}{c}\text { Quartile } 1 \text { (lowest) vs. } 2 \text { vs. } 3 \text { vs. } 4 \text {, female } \\
36.2 \% \text { vs. } 43.4 \% \text { vs. } 42.5 \% \text { vs. } 38.6 \%, p=0.517 \\
\text { (mean, } 6.76 \text { pmol/L) }\end{array}$ & Osteoprotegerin & [115] \\
\hline Jean et al. & France & 2011 & 5D (HD) & 1138 & Neutral & $\begin{array}{c}<50 \text { vs. } \geq 50 \mathrm{pg} / \mathrm{mL}, \text { female } 43 \% \text { vs. } 40 \%, p> \\
0.05\end{array}$ & Parathyroid hormone & [169] \\
\hline $\begin{array}{l}\text { González- } \\
\text { Parra E } \\
\text { et al. }\end{array}$ & Spain & 2015 & $1-5$ & 704 & $\begin{array}{l}\text { Female } \\
\text { higher in } \\
\text { both }\end{array}$ & $\begin{array}{l}\text { For PTH, male } \mathrm{r}=-0.084(-0.155 \sim-0.012), p \\
=0.0215 \\
\begin{array}{c}\text { For FGF-23, male } \mathrm{r}=-0.191 \\
(-0.301 \sim-0.080), p=0.0007\end{array}\end{array}$ & $\begin{array}{l}\text { Parathyroid hormone } \\
\text { and FGF-23 }\end{array}$ & [164] \\
\hline $\begin{array}{l}\text { Thambiah } \\
\text { et al. }\end{array}$ & $\begin{array}{l}\text { United } \\
\text { Kingdom }\end{array}$ & 2012 & 3B-4 & 77 & Male higher & $\begin{array}{l}\text { Male vs. female, mean } 50.5 \text { vs. } 34 \mathrm{pmol} / \mathrm{L}, p \\
\qquad \begin{array}{c}=0.015 \\
\text { Male } \beta=0.23, \mathrm{p}=0.024\end{array}\end{array}$ & Sclerostin & [186] \\
\hline Kuo et al. & Taiwan & 2019 & 5D (PD) & 89 & Male higher & $\begin{array}{c}<80.2 \text { vs. } \geq 80.2 \mathrm{pmol} / \mathrm{L}, \text { male } 36.4 \% \text { vs. } \\
62.2 \%, p=0.015 \\
\text { Male OR } 2.882(1.219-6.815), \mathrm{p}=0.016\end{array}$ & Sclerostin & [172] \\
\hline $\begin{array}{l}\text { Goncalves } \\
\text { et al. }\end{array}$ & Brazil & 2014 & 5D (HD) & 91 & Neutral & $\begin{array}{l}\text { High vs. low levels, male } 68.9 \% \text { vs. } 52.2 \%, p \\
=0.103 \\
\text { Regression model, male OR } 0.82(0.39-1.75) \\
\qquad p=0.62\end{array}$ & Sclerostin & [163] \\
\hline Viaene et al. & Belgium & 2013 & $5 \mathrm{D}(\mathrm{HD})$ & 100 & Neutral & Low vs. high, female $47 \%$ vs. $35 \%, p=0.2$ & Sclerostin & [188] \\
\hline Claes et al. & Belgium & 2013 & $1-5$ & 154 & Male higher & $\begin{array}{c}\text { Male gender significantly associated with } \\
\text { higher levels }(p=0.006)\end{array}$ & Sclerostin & [162] \\
\hline Zou et al. & China & 2020 & $5 \mathrm{D}$ & 165 & Male higher & $\begin{array}{l}\text { Among PD patients, male } \beta=0.259 \\
(0.052-0.416), p=0.012\end{array}$ & Sclerostin & [191] \\
\hline Jean et al. & France & 2016 & 5D (HD) & 227 & $\begin{array}{l}\text { Female } \\
\text { lower }\end{array}$ & Female OR $0.16(0.075-0.362)$ & Sclerostin & [66] \\
\hline Evenpoel et al. & Belgium & 2015 & $5 \mathrm{~T}$ & 268 & Male higher & $\begin{array}{c}\text { Male gender significantly associated with } \\
\text { higher levels }(p=0.002)\end{array}$ & Sclerostin & [130] \\
\hline Zhang et al. & China & 2019 & 5D (HD) & 105 & Neutral & $\begin{array}{c}\text { Quartile } 1 \text { (lowest) vs. } 2 \text { vs. } 3 \text { vs. } 4 \text {, male } \\
57.7 \% \text { vs. } 48.1 \% \text { vs. } 46.2 \% \text { vs. } 65.4 \%, p=0.473\end{array}$ & Soluble Klotho & [189] \\
\hline Buiten MS & Netherlands & 2014 & $5 \mathrm{D}$ & 127 & $\begin{array}{l}\text { Female } \\
\text { higher }\end{array}$ & $\begin{array}{c}<460 \text { vs. }>460 \mathrm{pg} / \mathrm{mL} \text {, female } 16 \% \text { vs. } 31 \%, p \\
<0.05\end{array}$ & Soluble Klotho & [159] \\
\hline Cai et al. & China & 2015 & 5D (HD) & 129 & Neutral & $\begin{array}{c}\leq 379 \text { vs. } 379-613 \text { vs. } 613-817 \text { vs. }>817 \\
\text { pg } / \mathrm{mL} \text {, male } 59.4 \% \text { vs. } 54.5 \% \text { vs. } 56.3 \% \text { vs. } \\
53.1 \%, p>0.05\end{array}$ & Soluble Klotho & [129] \\
\hline
\end{tabular}


Table 1. Cont.

\begin{tabular}{|c|c|c|c|c|c|c|c|c|}
\hline Authors & Country & Time & $\begin{array}{l}\text { CKD } \\
\text { Stages }\end{array}$ & $\begin{array}{l}\text { Sample } \\
\text { Size }\end{array}$ & $\begin{array}{l}\text { Gender } \\
\text { Effect }\end{array}$ & Data & Potential Modifiers & Ref. \\
\hline Jean et al. & France & 2008 & 5D (HD) & 253 & $\begin{array}{c}\text { Female } \\
\text { lower levels }\end{array}$ & $\begin{array}{l}\text { Deficient vs. sufficient, female } 53 \% \text { vs. } 28 \% \text {, } \\
\qquad p<0.05\end{array}$ & $\begin{array}{l}\text { Vitamin D (25-OH-D) } \\
\text { deficiency }\end{array}$ & [168] \\
\hline Chang et al. & $\begin{array}{l}\text { Republic of } \\
\text { Korea }\end{array}$ & 2012 & 5D (HD) & 289 & $\begin{array}{c}\text { Female } \\
\text { lower levels }\end{array}$ & Female OR $3.892(1.678-9.025), p=0.002$ & $\begin{array}{l}\text { Vitamin } \mathrm{D}(25-\mathrm{OH}-\mathrm{D}) \\
\text { deficiency } \\
(<15 \mathrm{ng} / \mathrm{mL})\end{array}$ & [36] \\
\hline \multicolumn{9}{|l|}{ Miscellaneous } \\
\hline Kato et al. & Japan & 2009 & 5D (HD) & 68 & Neutral & No correlation with gender & $\begin{array}{l}\text { Serum pre-B cell } \\
\text { colony-enhancing } \\
\text { factor/visfatin }\end{array}$ & [171] \\
\hline He et al. & China & 2018 & 5D (HD) & 150 & Neutral & Male correlation coefficient $0.106, p=0.114$ & Serum irisin & [59] \\
\hline Liabeuf et al. & France & 2013 & $2-5,5 \mathrm{D}$ & 139 & Neutral & $\begin{array}{c}\leq 0.041 \text { vs. }>0.041 \mathrm{mg} / \mathrm{dL}, \text { male } 63 \% \text { vs. } 57 \% \text {, } \\
p=0.5\end{array}$ & $\begin{array}{c}\text { Free } \\
\text { p-cresylglucuronide }\end{array}$ & [173] \\
\hline Ulusoy et al. & Turkey & 2012 & 5D (HD) & 103 & Male higher & $\begin{array}{c}\text { Pre-dialysis male vs. female levels, } \\
314.7 \pm 157.1 \text { vs. } 210.4 \pm 115.4 \mathrm{ng} / \mathrm{mL} \\
\qquad<<0.001\end{array}$ & $\begin{array}{l}\text { Signal } \\
\text { peptide-CUB-EGF } \\
\text { domain-containing } \\
\text { protein } 1 \text { (SCUBE1) }\end{array}$ & [187] \\
\hline Wang et al. & Hong Kong & 2014 & $3-5$ & 300 & Male lower & Male correlation coefficient $-0.14, p=0.02$ & $\begin{array}{c}\text { Tissue AGEs } \\
\text { (surrogated by skin } \\
\text { autofluorescence) }\end{array}$ & [107] \\
\hline
\end{tabular}

ADPN, adiponectin; AGE, advanced glycation endproduct; CRP, C-reactive protein; Dp-ucMGP, dephosphorylated-uncarboxylated Matrix Gla protein; ECF, extracellular fluid; FGF-23, fibroblast growth factor-23; HD, hemodialysis; HDL, high density lipoprotein; HMW, high molecular weight; HR, hazard ratio; LDL, low density lipoprotein; LMW, low molecular weight; MGP, matrix Gla protein; OR, odds ratio; PD, peritoneal dialysis.

\section{Overall Interpretations}

In this literature review involving 167 studies of CKD-associated VC, we found that the majority identified males with a higher VC prevalence/severity compared to females, although stages of CKD, the approaches used to measure VC, and the number of cases per study may account for the heterogeneity in study findings. In patients with non-dialysis CKD, males might not necessarily have a higher VC prevalence/severity, especially when studies assessed VC surrogates, except for those focused on CAC. In patients with dialysisdependent CKD, males still exhibited a higher VC risk, but heterogeneity persisted. Females from Asian countries exhibited a higher tendency of having carotid artery calcification relative to males. These findings appeared to be more uniform after multivariable adjustment, as studies in patients with non-dialysis dependent and dialysis-dependent CKD mostly supported a higher VC risk among males. Potential contributors to the observed gender-related differences can be multiple, including pericardial/total fat, lipid profile, inflammatory status, variations in MGP, soluble Klotho, vitamin D, sclerostin, PTH, FGF-23, and osteoprotegerin levels.

\subsection{Intimal or Non-Intimal Calcification: A Potential Consideration for Gender-Related Differences in CKD-Associated VC Risk}

Findings from our literature summary suggest that reports focusing on CAC more frequently identified a male preponderance in VC prevalence/risk (Supplementary Tables S1 and S2). Coronary atherosclerosis frequently begins with the retention of LDL and/or Lp(a) in subendothelial extracellular matrix, leading to local inflammation, cellular necrosis, and attraction of monocytes with phagocytosis of the degraded content. The necrotic core serves as the niche of calcification nucleation, assisted further by the enhanced release of pro-calcifying vesicles and the reduced expression of mineralization inhibitors by neighboring cells [194]. CAC is therefore deemed more akin to a symbol of atherosclerotic intimal calcification, although medial calcification exists simultaneously. On the other hand, calcifications involving smaller diameter vessels such as arteriovenous accesses, or digital arteries are more likely to be medially located and are pathophysiologically distinct from intimal type calcification [195]. Since male 
gender correlates with a higher risk of atherosclerosis and potentially atherosclerotic calcification [20], studies focusing on CAC may be inclined to derive the finding that males have an increased VC risk relative to females.

\subsection{Reports Favoring a Higher Risk of CKD-Associated VC in Males: Plausible Mediators}

We discovered that in CKD patients, especially those with non-dialysis status, males were at a higher risk of VC compared to females (Supplementary Table S2). It was further disclosed that males had a significantly higher proportion of pericardial fat and higher levels of serum ACE2, LDL, vitamin D, and sclerostin than females, but lower HDL, adiponectin, uncarboxylated MGP and soluble Klotho (Table 1). Prior studies showed that a higher amount of pericardial fat tissues was associated with a greater CAC severity at baseline and a higher speed of CAC progression, through their paracrine and endocrine effects of inducing inflammation, oxidative stress, and endothelial dysfunction [196]. A study disclosed that aortic ACE2 expression was significantly up-regulated in calcified aortas [197], and ACE2 presumably plays an important role in perpetuating local inflammation. Calcitriol supplementations have been reported to intensify the severity and accelerate the tempo of VC in models of CKD and DM [198,199], suggesting that differences in vitamin D levels may be partially responsible for gender-related differences in VC risk. Sclerostin, an inhibitor of the canonical Wnt/ $\beta$-catenin pathway originally thought to be solely expressed in bones, is found to be up-regulated in VSMCs and serves as a predisposing factor for VC [200]. It is likely that higher expressions of sclerostin in male CKD patients contribute to their tendency of developing VC. Along the same line, anti-calcific factors in CKD such as MGP and Klotho [201,202] were also lower in male CKD patients (Table 1), further paving the ground for their pro-calcific tendency. Dyslipidemia, in the forms of high LDL or low $\mathrm{HDL}$, is associated with an increased risk of VC progression through their pathogenic linkage to atherosclerosis and local inflammation $[203,204]$. From this perspective, male CKD patients may harbor several risk features that predispose themselves to VC development.

\subsection{Reports Favoring Higher Risk of CKD-Associated VC in Females: Clinical Implications and Plausible Mediators}

Few studies (5\% to 7\%) in our summary identified females as having an increased VC risk relative to males (Supplementary Tables S1 and S2), while they predominantly focused on aortic or carotid artery calcification and tended to originate from Asian countries. Experimental reports showed that through binding to estrogen receptor $\alpha$, estrogen could inhibit receptor activator of nuclear factor $\mathrm{k}$ ligand (RANKL) signaling, leading to VSMC trans-differentiation and calcification [205]. However, there are also studies suggesting that estrogen analogs could aggravate VSMC calcification [206]. These findings indicate that the influence of estrogen on VC can be complex and scenario-dependent, unlike that exerted by testosterone, which is consistently pro-calcific [207]. Several other features can further modify these gender-related differences in VC risk, including the higher serum phosphate, FGF-23, and PTH levels observed in female CKD patients (Table 1). Elevated levels of FGF-23 and PTH parallel the worsening of CKD-associated VC in clinical studies, and participate in VC pathogenesis according to experimental reports [208]. Consequently, alterations in these hormones may be responsible for the increased risk of CKD-associated VC in females, at least partially.

\subsection{Gender-Related Differences in Osteoprotegerin Levels and CKD-Associated VC}

We observed substantial heterogeneity in studies reporting gender-related differences in serum osteoprotegerin levels focusing on $\mathrm{VC}$; there were reports yielding no genderrelated difference, while others reported either higher levels in males or in females (Table 1). It is clear that studies enrolling more patient numbers $(>300)$ tend to identify higher osteoprotegerin levels in females but not males with CKD. In vitro studies indicated that estrogen could stimulate the up-regulation of osteoprotegerin in osteoblasts, but the direction of effect may depend on the types of estrogen receptors [209]. Consequently, clinical findings that higher serum osteoprotegerin levels alternated between different genders are 
not unexpected, and a careful interpretation of results in the context of sample size and other influencing factors is needed.

\section{Conclusions}

Gender-related differences in various biological phenotypes do exist, and such differences greatly affect cardiovascular risk estimation and carry public health importance. The male gender is traditionally regarded as a risk trait for atherosclerosis, but whether this relationship applies to VC remains debatable. VC encompasses a complex molecular interplay between endothelial cells, VSMCs, adventitial fibroblasts, pericytes, and even infiltrating macrophages, further compounded by the influences of uremic milieu and pro-/anti-calcific factors. We systematically identified existing reports of CKD-associated VC or its potential regulators containing data about gender distributions, and synthesizing their messages. Though a higher risk of CKD-associated VC risk was observed in males among the majority of original investigations, heterogeneity in findings were found; multiple large-scale studies yielded neutral findings, and few reported a higher risk of CKD-associated VC among females. Clinically speaking, differences in findings of gender-related VC risk may result from variations in outcome assessment approaches, the anatomical segments of interest, sample size, and even ethnic origins of participants. Biologically speaking, plausible mediators of such gender-related difference may include body composition changes, lipid profiles, inflammatory status, and diversities in MGP, soluble Klotho, vitamin D, sclerostin, PTH, FGF-23, and even osteoprotegerin levels. Based on our findings, it may not be appropriate to assign a high-risk VC category to males with CKD, and more background in context should be contemplated before reaching conclusions.

Supplementary Materials: The following are available online at https:/ / www.mdpi.com/article / 10.3390/healthcare9080979/s1, Table S1: Gender-related differences in the prevalence and/or severity of vascular calcification in existing studies, Table S2: Gender-related adjusted risk of vascular calcification in existing studies.

Author Contributions: Study design: P.Y.W. and C.-T.C.; data analysis: P.Y.W. and C.-T.C.; article drafting: P.Y.W., S.-Y.L., K.-V.C., C.-T.C. and J.-W.H. All authors have read and agreed to the published version of the manuscript.

Funding: The study is financially sponsored by the National Taiwan University Hospital BeiHu Branch (11001), the National Taiwan University Hospital Yunlin Branch, and the Ministry of Science and Technology, Taiwan (MOST 109-2314-B-002-193-MY3).

Institutional Review Board Statement: Not applicable.

Informed Consent Statement: Not applicable.

Data Availability Statement: The raw data for conducting this analysis are available upon reasonable request to the corresponding author and the first author.

Acknowledgments: We are grateful to the Second Core Laboratory, Department of Medical Research of the National Taiwan University Hospital and the National Taiwan University Center of Genomic and Precision Medicine for their technical input.

Conflicts of Interest: None of the authors has relevant financial or non-financial competing interests to declare in relation to this article. The sponsors have no role in the study design, data collection, analysis, and result interpretation of this study.

\section{References}

1. Yano, Y.; O’Donnell, C.J.; Kuller, L.; Kavousi, M.; Erbel, R.; Ning, H.; D’Agostino, R.; Newman, A.B.; Nasir, K.; Hofman, A.; et al. Association of Coronary Artery Calcium Score vs. Age with Cardiovascular Risk in Older Adults: An Analysis of Pooled Population-Based Studies. JAMA Cardiol. 2017, 2, 986-994. [CrossRef]

2. Chao, C.-T.; Han, D.-S.; Huang, J.-W. Circulating microRNA-125b Levels Are Associated with the Risk of Vascular Calcification in Healthy Community-Dwelling Older Adults. Front. Cardiovasc. Med. 2021, 8, 624313. [CrossRef] [PubMed] 
3. Miedema, M.D.; Dardari, Z.A.; Nasir, K.; Blankstein, R.; Knickelbine, T.; Oberembt, S.; Shaw, L.; Rumberger, J.; Michos, E.D.; Rozanski, A.; et al. Association of Coronary Artery Calcium with Long-term, Cause-Specific Mortality Among Young Adults. JAMA Netw. Open 2019, 2, e197440. [CrossRef] [PubMed]

4. Wang, X.-R.; Zhang, J.-J.; Xu, X.-X.; Wu, Y.-G. Prevalence of coronary artery calcification and its association with mortality, cardiovascular events in patients with chronic kidney disease: A systematic review and meta-analysis. Ren. Fail. 2019, 41, 244-256. [CrossRef]

5. Mencke, R.; van der Vaart, A.; Pasch, A.; Harms, G.; Waanders, F.; Bilo, H.J.G.; van Goor, H.; Hillebrands, J.-L.; van Dijk, P.R. Serum calcification propensity is associated with HbA1c in type 2 diabetes mellitus. BMJ Open Diabetes Res. Care 2021, 9 , e002016. [CrossRef] [PubMed]

6. Fakhry, M.; Sidhu, M.S.; Bangalore, S.; Mathew, R.O. Accelerated and intensified calcific atherosclerosis and microvascular dysfunction in patients with chronic kidney disease. Rev. Cardiovasc. Med. 2020, 21, 157-162. [CrossRef]

7. Lee, J.H.; Rizvi, A.; Hartaigh, B.Ó.; Han, D.; Park, M.W.; Roudsari, H.M.; Stuijfzand, W.J.; Gransar, H.; Lu, Y.; Callister, T.Q.; et al. The Predictive Value of Coronary Artery Calcium Scoring for Major Adverse Cardiac Events According to Renal Function (from the Coronary Computed Tomography Angiography Evaluation for Clinical Outcomes: An International Multicenter [CONFIRM] Registry). Am. J. Cardiol. 2019, 123, 1435-1442. [CrossRef] [PubMed]

8. $\quad$ Eelderink, C.; Te Velde-Keyzer, C.A.; Frenay, A.S.; Vermeulen, E.A.; Bachtler, M.; Aghagolzadeh, P.; van Dijk, P.R.; Gansevoort, R.T.; Vervloet, M.G.; Hillebrands, J.-A.; et al. Serum Calcification Propensity and the Risk of Cardiovascular and All-Cause Mortality in the General Population: The PREVEND Study. Arterioscler. Thromb. Vasc. Biol. 2020, 40, 1942-1951. [CrossRef] [PubMed]

9. Cano-Megías, M.; Guisado-Vasco, P.; Bouarich, H.; de Arriba-de la Fuente, G.; De Sequera-Ortiz, P.; Álvarez-Sanz, C.; RodríguezPuyol, D. Coronary calcification as a predictor of cardiovascular mortality in advanced chronic kidney disease: A prospective long-term follow-up study. BMC Nephrol. 2019, 20, 188. [CrossRef]

10. Wang, Z.; Jiang, A.; Wei, F.; Chen, H. Cardiac valve calcification and risk of cardiovascular or all-cause mortality in dialysis patients: A meta-analysis. BMC Cardiovasc. Disord. 2018, 18, 12. [CrossRef]

11. Chen, S.-C.; Teh, M.; Huang, J.-C.; Wu, P.-Y.; Chen, C.-Y.; Tsai, Y.-C.; Chiu, Y.-W.; Chang, J.-M.; Chen, H.-C. Increased Aortic Arch Calcification and Cardiomegaly is Associated with Rapid Renal Progression and Increased Cardiovascular Mortality in Chronic Kidney Disease. Sci. Rep. 2019, 9, 1-9. [CrossRef]

12. Handy, C.E.; Desai, C.S.; Dardari, Z.A.; Al-Mallah, M.H.; Miedema, M.D.; Ouyang, P.; Budoff, M.J.; Blumenthal, R.S.; Nasir, K.; Blaha, M.J. The Association of Coronary Artery Calcium with Noncardiovascular Disease: The Multi-Ethnic Study of Atherosclerosis. JACC Cardiovasc. Imaging 2016, 9, 568-576. [CrossRef] [PubMed]

13. Lee, S.; Chao, C.; Huang, J.; Huang, K. Vascular Calcification as an Underrecognized Risk Factor for Frailty in 1783 CommunityDwelling Elderly Individuals. J. Am. Hear. Assoc. 2020, 9. [CrossRef] [PubMed]

14. Chao, C.-T.; Yeh, H.-Y.; Tsai, Y.-T.; Chuang, P.-H.; Yuan, T.-H.; Huang, J.-W.; Chen, H.-W. Natural and non-natural antioxidative compounds: Potential candidates for treatment of vascular calcification. Cell Death Discov. 2019, 5, 145. [CrossRef]

15. Hou, Y.-C.; Lu, C.-L.; Yuan, T.-H.; Liao, M.-T.; Chao, C.-T.; Lu, K.-C. The Epigenetic Landscape of Vascular Calcification: An Integrative Perspective. Int. J. Mol. Sci. 2020, 21, 980. [CrossRef]

16. Tsai, Y.-T.; Yeh, H.-Y.; Chao, C.-T.; Chiang, C.-K. Superoxide Dismutase 2 (SOD2) in Vascular Calcification: A Focus on Vascular Smooth Muscle Cells, Calcification Pathogenesis, and Therapeutic Strategies. Oxidative Med. Cell. Longev. 2021, 2021, 1-9. [CrossRef] [PubMed]

17. Disthabanchong, S.; Srisuwarn, P. Mechanisms of Vascular Calcification in Kidney Disease. Adv. Chronic Kidney Dis. 2019, 26, 417-426. [CrossRef]

18. Chao, C.; Yeh, H.; Yuan, T.; Chiang, C.; Chen, H. MicroRNA-125b in vascular diseases: An updated systematic review of pathogenetic implications and clinical applications. J. Cell. Mol. Med. 2019, 23, 5884-5894. [CrossRef]

19. Chen, Y.; Zhao, X.; Wu, H. Arterial Stiffness: A Focus on Vascular Calcification and Its Link to Bone Mineralization. Arterioscler. Thromb. Vasc. Biol. 2020, 40, 1078-1093. [CrossRef] [PubMed]

20. Calabrò, P.; Niccoli, G.; Gragnano, F.; Grove, E.L.; Vergallo, R.; Mikhailidis, D.P.; Patti, G.; Spaccarotella, C.; Katsiki, N.; Masiero, G.; et al. Are we ready for a gender-specific approach in interventional cardiology? Int. J. Cardiol. 2019, 286, 226-233. [CrossRef]

21. Tani, S.; Matsuo, R.; Imatake, K.; Suzuki, Y.; Yagi, T.; Takahashi, A.; Matsumoto, N.; Okumura, Y. Gender differences in the associations among fish intake, lifestyle, and non-HDL-C level in Japanese subjects over the age of 50 years: Anti-atherosclerotic effect of fish consumption. Nutr. Metab. Cardiovasc. Dis. 2021, 31, 1434-1444. [CrossRef]

22. Bairey Merz, C.N.; Shaw, L.J.; Reis, S.E.; Bittner, V.; Kelsey, S.F.; Olson, M.; Johnson, B.D.; Pepine, C.J.; Mankad, S.; Sharaf, B.L.; et al. Insights from the NHLBI-Sponsored Women's Ischemia Syndrome Evaluation (WISE) Study: Part II: Gender differences in presentation, diagnosis, and outcome with regard to gender-based pathophysiology of atherosclerosis and macrovascular and microvascular coronary disease. J. Am. Coll. Cardiol. 2006, 47 (Suppl. 3), S21-S29.

23. Tao, X.-Y.; Zuo, A.-Z.; Wang, J.-Q.; Tao, F.-B. Effect of primary ovarian insufficiency and early natural menopause on mortality: A meta-analysis. Climacteric 2016, 19, 27-36. [CrossRef] [PubMed]

24. Kim, B.S.; Chan, N.; Hsu, G.; Makaryus, A.N.; Chopra, M.; Cohen, S.L.; Makaryus, J.N. Sex Differences in Coronary Arterial Calcification in Symptomatic Patients. Am. J. Cardiol. 2021, 149, 16-20. [CrossRef] 
25. Inker, L.A.; Astor, B.C.; Fox, C.H.; Isakova, T.; Lash, J.P.; Peralta, C.A.; Tamura, M.K.; Feldman, H.I. KDOQI US Commentary on the 2012 KDIGO Clinical Practice Guideline for the Evaluation and Management of CKD. Am. J. Kidney Dis. 2014, 63, 713-735. [CrossRef]

26. Ahmed, S.; Neill, K.D.O.; Hood, A.F.; Evan, A.P.; Moe, S.M. Calciphylaxis is associated with hyperphosphatemia and increased osteopontin expression by vascular smooth muscle cells. Am. J. Kidney Dis. 2001, 37, 1267-1276. [CrossRef] [PubMed]

27. Alayoud, A.; El Amrani, M.; Belarbi, M.; El Kharras, A.; Chtioui, M.; Elfilali, K. Facteurs de risque de progression des calcifications des artères coronaires après 5 ans d'évolution en dialyse. Ann. Cardiol. d'Angéiol. 2020, 69, 81-85. [CrossRef] [PubMed]

28. Al-Rifai, R.; Arabi, A.; Masrouji, R.; Daouk, M. Prevalence of peripheral vascular calcifications in patients on chronic hemodialysis at a tertiary care center in Beirut: A pilot study. Leban. Med. J. 2011, 59, 117-121.

29. Asci, G.; Ok, E.; Savas, R.; Ozkahya, M.; Duman, S.; Töz, H.; Kayikcioglu, M.; Branscum, A.J.; Monier-Faugere, M.-C.; Herberth, J.; et al. The link between bone and coronary calcifications in CKD-5 patients on haemodialysis. Nephrol. Dial. Transplant. 2011, 26, 1010-1015. [CrossRef] [PubMed]

30. Avramovski, P.; Avramovska, M.; Sotiroski, K.; Sikole, A. Acute-phase proteins as promoters of abdominal aortic calcification in chronic dialysis patients. Saudi J. Kidney Dis. Transplant. 2019, 30, 376-386. [CrossRef]

31. Bae, E.; Seong, E.Y.; Han, B.-G.; Kim, D.K.; Lim, C.S.; Kang, S.-W.; Park, C.W.; Kim, C.-D.; Shin, B.C.; Kim, S.G.; et al. Coronary artery calcification in Korean patients with incident dialysis. Hemodial. Int. 2017, 21, 367-374. [CrossRef]

32. Ballotta, E.; Renon, L.; Toffano, M.; Piccoli, A.; Da Giau, G. Patency and limb salvage rates after distal revascularization to unclampable calcified outflow arteries. J. Vasc. Surg. 2004, 39, 539-546. [CrossRef] [PubMed]

33. Bellasi, A.; Block, G.A.; Ferramosca, E.; Ratti, C.; Raggi, P. Integration of clinical and imaging data to predict death in hemodialysis patients. Hemodial. Int. 2013, 17, 12-18. [CrossRef]

34. Bundy, J.D.; Cai, X.; Mehta, R.C.; Scialla, J.J.; De Boer, I.H.; Hsu, C.-Y.; Go, A.S.; Dobre, M.A.; Chen, J.; Rao, P.S.; et al. Serum Calcification Propensity and Clinical Events in CKD. Clin. J. Am. Soc. Nephrol. 2019, 14, 1562-1571. [CrossRef]

35. Rao, N.; Chandra, A.; Raj, G.; Awasthi, N.P.; Srivastava, D. Evaluation of the relationship between blood cell parameters and vascular calcification in dialysis-dependent end-stage renal disease patients. Saudi J. Kidney Dis. Transplant. 2020, 31, 136-143. [CrossRef] [PubMed]

36. Chang, J.H.; Ro, H.; Kim, S.; Lee, H.H.; Chung, W.; Jung, J.Y. Study on the relationship between serum 25-hydroxyvitamin D levels and vascular calcification in hemodialysis patients with consideration of seasonal variation in vitamin D levels. Atherosclerosis 2012, 220, 563-568. [CrossRef] [PubMed]

37. Chao, C.-T.; Yeh, H.-Y.; Tsai, Y.-T.; Chiang, C.-K.; Chen, H.-W. A combined microRNA and target protein-based panel for predicting the probability and severity of uraemic vascular calcification: A translational study. Cardiovasc. Res. 2021, 117, 1958-1973. [CrossRef]

38. Chao, C.-T.; Liu, Y.-P.; Su, S.-F.; Yeh, H.-Y.; Chen, H.-Y.; Lee, P.-J.; Chen, W.-J.; Lee, Y.-M.; Huang, J.-W.; Chiang, C.-K.; et al. Circulating MicroRNA-125b Predicts the Presence and Progression of Uremic Vascular Calcification. Arter. Thromb. Vasc. Biol. 2017, 37, 1402-1414. [CrossRef]

39. Charitaki, E.; Davenport, A. Aortic pulse wave velocity in haemodialysis patients is associated with the prescription of active vitamin D analogues. J. Nephrol. 2014, 27, 431-437. [CrossRef]

40. Chen, Z.; Qureshi, A.R.; Parini, P.; Hurt-Camejo, E.; Ripsweden, J.; Brismar, T.; Barany, P.; Jaminon, A.M.; Schurgers, L.; Heimbürger, O.; et al. Does statins promote vascular calcification in chronic kidney disease? Eur. J. Clin. Investig. 2017, 47, 137-148. [CrossRef] [PubMed]

41. Chen, J.; Budoff, M.J.; Reilly, M.; Yang, W.; Rosas, S.E.; Rahman, M.; Zhang, X.; Roy, J.A.; Lustigova, E.; Nessel, L.; et al. Coronary Artery Calcification and Risk of Cardiovascular Disease and Death Among Patients with Chronic Kidney Disease. JAMA Cardiol. 2017, 2, 635-643. [CrossRef] [PubMed]

42. Chiu, Y.-W.; Adler, S.G.; Budoff, M.J.; Takasu, J.; Ashai, J.; Mehrotra, R. Coronary artery calcification and mortality in diabetic patients with proteinuria. Kidney Int. 2010, 77, 1107-1114. [CrossRef] [PubMed]

43. Choi, S.R.; Lee, Y.-K.; Cho, A.J.; Park, H.C.; Han, C.H.; Choi, M.-J.; Koo, J.-R.; Yoon, J.-W.; Noh, J.W. Malnutrition, inflammation, progression of vascular calcification and survival: Inter-relationships in hemodialysis patients. PLoS ONE 2019, 14, e0216415. [CrossRef]

44. Chue, C.D.; Wall, N.A.; Crabtree, N.J.; Zehnder, D.; Moody, W.E.; Edwards, N.C.; Steeds, R.; Townend, J.N.; Ferro, C.J. Aortic Calcification and Femoral Bone Density Are Independently Associated with Left Ventricular Mass in Patients with Chronic Kidney Disease. PLoS ONE 2012, 7, e39241. [CrossRef]

45. Claes, K.J.; Heye, S.; Bammens, B.; Kuypers, D.R.; Meijers, B.; Naesens, M.; Vanrenterghem, Y.; Evenepoel, P. Aortic calcifications and arterial stiffness as predictors of cardiovascular events in incident renal transplant recipients. Transpl. Int. 2013, 26, 973-981. [CrossRef] [PubMed]

46. Coen, G.; Manni, M.; Agnoli, A.; Balducci, A.; Dessi, M.; De Angelis, S.; Jankovic, L.; Mantella, D.; Morosetti, M.; Naticchia, A.; et al. Cardiac Calcifications: Fetuin???A and Other Risk Factors in Hemodialysis Patients. ASAIO J. 2006, 52, 150-156. [CrossRef]

47. Coll, B.; Betriu, A.; Martinez-Alonso, M.; Amoedo, M.L.; Arcidiacono, M.V.; Borras, M.; Valdivielso, J.M.; Fernández, E. Large Artery Calcification on Dialysis Patients Is Located in the Intima and Related to Atherosclerosis. Clin. J. Am. Soc. Nephrol. 2011, 6, 303-310. [CrossRef] 
48. Craver, L.; Dusso, A.; Martinez-Alonso, M.; Sarro, F.; Valdivielso, J.M.; Fernández, E. A low fractional excretion of Phosphate/Fgf23 ratio is associated with severe abdominal Aortic calcification in stage 3 and 4 kidney disease patients. BMC Nephrol. 2013, 14, 1-221. [CrossRef]

49. Davis, B.; Marin, D.; Hurwitz, L.M.; Ronald, J.; Ellis, M.J.; Ravindra, K.V.; Collins, B.H.; Kim, C.Y. Application of a Novel CT-Based Iliac Artery Calcification Scoring System for Predicting Renal Transplant Outcomes. Am. J. Roentgenol. 2016, 206, $436-441$. [CrossRef]

50. Deloach, S.S.; Joffe, M.M.; Mai, X.; Goral, S.; Rosas, S.E. Aortic calcification predicts cardiovascular events and all-cause mortality in renal transplantation. Nephrol. Dial. Transplant. 2009, 24, 1314-1319. [CrossRef]

51. Di Iorio, B.R.; Bortone, S.; Piscopo, C.; Grimaldi, P.; Cucciniello, E.; D’Avanzo, E.; Ernesto, A.; Fiorentino, M.; Nicola, C.; Vincenzoet, B. Cardiac Vascular Calcification and QT Interval in ESRD Patients: Is There a Link? Blood Purificat. 2006, 24, 451-459. [CrossRef] [PubMed]

52. Disthabanchong, S.; Vipattawat, K.; Phakdeekitcharoen, B.; Kitiyakara, C.; Sumethkul, V. Abdominal aorta and pelvic artery calcifications on plain radiographs may predict mortality in chronic kidney disease, hemodialysis and renal transplantation. Int. Urol. Nephrol. 2018, 50, 355-364. [CrossRef] [PubMed]

53. El Amrani, M.; Maoujoud, O.; Belarbi, M.; El Farouki, M.R.; Zajjari, Y.; Boukili, Y.; Bouzelmate, H.; Rbaibi, A.; El Kharras, A.; Salahedine, T.; et al. Dépistage et facteurs de risque des calcifications cardiaques chez l'hémodialysé: Apport du scanner multi-coupe ultra-rapide et de l'échocardiographie transthoracique. Ann. Cardiol. d'Angéiol. 2015, 64, 87-93. [CrossRef] [PubMed]

54. Etta, P.K.; Sharma, R.K.; Gupta, A. Study of chronic kidney disease-mineral bone disorders in newly detected advanced renal failure patients: A Hospital-based cross-sectional study. Saudi J. Kidney Dis. Transplant. 2017, 28, 874-885.

55. Fabbian, F.; Catalano, C.; Orlandi, V.; Conte, M.M.; Lupo, A.; Catizone, L. Evaluation of aortic arch calcification in hemodialysis patients. J. Nephrol. 2005, 18, 289-293.

56. Fayed, A.; Soliman, A.; El Mahdy, H.; Hamza, W.; Abdulazim, D.O.; Salem, M.M.; El Din, U.A.S.; on behalf of the Vascular Calcification Group (VCG). Intraoperative Arterial Biopsy in Incident Hemodialysis Patients: Differences Observed. Nephron 2019, 143, 54-61. [CrossRef]

57. Gunen Yilmaz, S.; Yilmaz, F.; Bayrakdar, I.; Harorli, A. The Relationship between carotid artery calcification and pulp stone among hemodialysis patients: A retrospective study. Saudi J. Kidney Dis. Transplant. 2019, 30, 755-763. [CrossRef] [PubMed]

58. Harada, P.H.N.; Canziani, M.E.F.; Lima, L.M.; Kamimura, M.A.; Rochitte, C.E.; Lemos, M.M.; Cuppari, L.; Filho, R.K.; Draibe, S.A.; Santos, R.D. Pericardial Fat Is Associated with Coronary Artery Calcification in Non-Dialysis Dependent Chronic Kidney Disease Patients. PLoS ONE 2014, 9, e114358. [CrossRef]

59. He, L.; He, W.-Y.; La-Ta, A.; Yang, W.-L.; Zhang, A.-H. Lower Serum Irisin Levels Are Associated with Increased Vascular Calcification in Hemodialysis Patients. Kidney Blood Press. Res. 2018, 43, 287-295. [CrossRef]

60. He, J.; Reilly, M.; Yang, W.; Chen, J.; Go, A.S.; Lash, J.P.; Rahman, M.; DeFilippi, C.; Gadegbeku, C.; Kanthety, R.; et al. Risk Factors for Coronary Artery Calcium Among Patients with Chronic Kidney Disease (from the Chronic Renal Insufficiency Cohort Study). Am. J. Cardiol. 2012, 110, 1735-1741. [CrossRef] [PubMed]

61. Hou, J.-S.; Lin, Y.-L.; Wang, C.-H.; Lai, Y.-H.; Kuo, C.-H.; Subeq, Y.-M.; Hsu, B.-G. Serum osteoprotegerin is an independent marker of central arterial stiffness as assessed using carotid-femoral pulse wave velocity in hemodialysis patients: A cross sectional study. BMC Nephrol. 2019, 20, 184. [CrossRef]

62. Al Humoud, H.; Al-Hilali, N.; Ahmad, A.A.M.H.; Ninan, V.T.; Nampoory, M.R.N.; Rizk, A.M.; Ali, J.H.; Johny, K.V. Vascular Calcification in Dialysis Patients. Transplant. Proc. 2005, 37, 4183-4186. [CrossRef]

63. Jankovic, A.; Damjanovic, T.; Djuric, Z.; Marinkovic, J.; Schlieper, G.; Djuric, P.; Dragovic, J.T.; Bulatovic, A.; Mitrovic, M.; Popovic, J.; et al. Calcification in arteriovenous fistula blood vessels may predict arteriovenous fistula failure: A 5-year follow-up study. Int. Urol. Nephrol. 2017, 49, 881-887. [CrossRef]

64. Janković, A.; Damjanovic, T.; Djuric, Z.; Marinkovic, J.; Schlieper, G.; Tosic-Dragovic, J.; Djuric, P.; Popovic, J.; Floege, J.; Dimkovic, N. Impact of Vascular Calcifications on Arteriovenous Fistula Survival in Hemodialysis Patients: A Five-Year Follow-Up. Nephron 2015, 129, 247-252. [CrossRef] [PubMed]

65. Jansson, H.; Saeed, A.; Svensson, M.K.; Finnved, K.; Hellström, M.; Guron, G. Impact of Abdominal Aortic Calcification on Central Haemodynamics and Decline of Glomerular Filtration Rate in Patients with Chronic Kidney Disease Stages 3 and 4. Kidney Blood Press. Res. 2019, 44, 950-960. [CrossRef]

66. Jean, G.; Chazot, C.; Bresson, E.; Zaoui, E.; Cavalier, E. High Serum Sclerostin Levels Are Associated with a Better Outcome in Haemodialysis Patients. Nephron 2016, 132, 181-190. [CrossRef]

67. Jean, G.; Bresson, E.; Terrat, J.-C.; Vanel, T.; Hurot, J.-M.; Lorriaux, C.; Mayor, B.; Chazot, C. Peripheral vascular calcification in long-haemodialysis patients: Associated factors and survival consequences. Nephrol. Dial. Transplant. 2009, 24, 948-955. [CrossRef] [PubMed]

68. Jean, G.; Bresson, É.; Lorriaux, C.; Mayor, B.; Hurot, J.-M.; Deleaval, P.; Chazot, C. Increased Levels of Serum Parathyroid Hormone and Fibroblast Growth Factor-23 Are the Main Factors Associated with the Progression of Vascular Calcification in Long-Hour Hemodialysis Patients. Nephron 2012, 120, c132-c138. [CrossRef] [PubMed]

69. Jiménez Villodres, M.; García Gutiérrez, G.; García Frías, P.; Rioja Villodres, J.; Martín Velázquez, M.; Sánchez Chaparro, M.Á.; López, C.P.; Valdivielso, P. Fractional excretion of phosphorus and vascular calcification in stage 3 chronic kidney disease. J. Investig. Med. 2019, 67, 674. [CrossRef] 
70. Kahn, J.; Ram, L.M.; Eberhard, K.; Müller, H.; Groselj-Strele, A.; Obermayer-Pietsch, B. Calcification score evaluation in patients listed for renal transplantation. Clin. Transplant. 2017, 31, e12888. [CrossRef] [PubMed]

71. Keyzer, C.A.; De Borst, M.; Berg, E.V.D.; Jahnen-Dechent, W.; Arampatzis, S.; Farese, S.; Bergmann, I.P.; Floege, J.; Navis, G.; Bakker, S.J.; et al. Calcification Propensity and Survival among Renal Transplant Recipients. J. Am. Soc. Nephrol. 2016, 27, 239-248. [CrossRef]

72. Kim, S.M.; Jung, I.M.; Kim, D.; Lee, J.P.; So, Y.H. Effect of Inflow Arterial Calcification on Arteriovenous Fistula Maturation. Ann. Vasc. Surg. 2019, 58, 331-337. [CrossRef]

73. Kim, H.G.; Song, S.W.; Kim, T.Y.; Kim, Y.O. Risk factors for progression of aortic arch calcification in patients on maintenance hemodialysis and peritoneal dialysis. Hemodial. Int. 2011, 15, 460-467. [CrossRef]

74. Kimura, K.; Saika, Y.; Otani, H.; Fujii, R.; Mune, M.; Yukawa, S. Factors associated with calcification of the abdominal aorta in hemodialysis patients. Kidney Int. 1999, 56, S238-S241. [CrossRef]

75. Komatsu, M.; Okazaki, M.; Tsuchiya, K.; Kawaguchi, H.; Nitta, K. Aortic Arch Calcification Predicts Cardiovascular and All-Cause Mortality in Maintenance Hemodialysis Patients. Kidney Blood Press. Res. 2014, 39, 658-667. [CrossRef]

76. Lee, C.-T.; Huang, C.-C.; Hsu, C.-Y.; Chiou, T.T.-Y.; Ng, H.-Y.; Wu, C.-H.; Kuo, W.-H.; Lee, Y.-T. Calcification of the Aortic Arch Predicts Cardiovascular and All-Cause Mortality in Chronic Hemodialysis Patients. Cardiorenal Med. 2014, 4, 34-42. [CrossRef] [PubMed]

77. Lee, C.-T.; Lee, Y.-T.; Tain, Y.-L.; Ng, H.-Y.; Kuo, W.-H. Circulating microRNAs and vascular calcification in hemodialysis patients. J. Int. Med. Res. 2019, 47, 2929-2939. [CrossRef] [PubMed]

78. Lee, M.J.; Park, J.T.; Park, K.S.; Kwon, Y.E.; Han, S.H.; Kang, S.-W.; Choi, K.H.; Oh, K.-H.; Park, S.K.; Chae, D.W.; et al. Normal body mass index with central obesity has increased risk of coronary artery calcification in Korean patients with chronic kidney disease. Kidney Int. 2016, 90, 1368-1376. [CrossRef]

79. Lioufas, N.M.; Pedagogos, E.; Hawley, C.M.; Pascoe, E.M.; Elder, G.J.; Badve, S.; Valks, A.; Toussaint, N.D.; on behalf of the IMPROVE-CKD Investigators. Aortic Calcification and Arterial Stiffness Burden in a Chronic Kidney Disease Cohort with High Cardiovascular Risk: Baseline Characteristics of the Impact of Phosphate Reduction On Vascular End-Points in Chronic Kidney Disease Trial. Am. J. Nephrol. 2020, 51, 201-215. [CrossRef] [PubMed]

80. Liu, J.; Zhang, L.; Zhou, Y.; Zhu, D.; Wang, Q.; Hao, L. Aberrant activation of Wnt pathways in arteries associates with vascular calcification in chronic kidney disease. Int. Urol. Nephrol. 2016, 48, 1313-1319. [CrossRef]

81. Lockhart, M.E.; Robbin, M.L.; McNamara, M.M.; Allon, M. Association of pelvic arterial calcification with arteriovenous thigh graft failure in haemodialysis patients. Nephrol. Dial. Transplant. 2004, 19, 2564-2569. [CrossRef]

82. London, G.M.; Pannier, B.; Marchais, S.J. Vascular Calcifications, Arterial Aging and Arterial Remodeling in ESRD. Blood Purif. 2013, 35, 16-21. [CrossRef] [PubMed]

83. Maharem, D.A.; Gomaa, S.H.; El Ghandor, M.K.; Mohamed, E.I.; Matrawy, K.A.; Zaytoun, S.S.; Nomeir, H.M. Association of serum fetuin-A and fetuin-A gene polymorphism in relation to mineral and bone disorders in patients with chronic kidney disease. Egypt. J. Med. Hum. Genet. 2013, 14, 337-352. [CrossRef]

84. Mazzaferro, S.; Pasquali, M.; Pugliese, F.; Barresi, G.; Carbone, I.; Francone, M.; Sardella, D.; Taggi, F. Serum Levels of Calcification Inhibition Proteins and Coronary Artery Calcium Score: Comparison between Transplantation and Dialysis. Am. J. Nephrol. 2007, 27, 75-83. [CrossRef]

85. Merjanian, R.; Budoff, M.; Adler, S.; Berman, N.; Mehrotra, R. Coronary artery, aortic wall, and valvular calcification in nondialyzed individuals with type 2 diabetes and renal disease. Kidney Int. 2003, 64, 263-271. [CrossRef] [PubMed]

86. Miyatake, N.; Adachi, H.; Nomura-Nakayama, K.; Okada, K.; Okino, K.; Hayashi, N.; Fujimoto, K.; Furuichi, K.; Yokoyama, H. Circulating CTRP9 correlates with the prevention of aortic calcification in renal allograft recipients. PLoS ONE 2020, 15, e0226526. [CrossRef]

87. Mizuiri, S.; Nishizawa, Y.; Yamashita, K.; Mizuno, K.; Ishine, M.; Doi, S.; Masaki, T.; Shigemoto, K. Coronary artery calcification score and common iliac artery calcification score in non-dialysis CKD patients. Nephrology 2018, 23, 837-845. [CrossRef] [PubMed]

88. Morena, M.; Dupuy, A.-M.; Jaussent, I.; Vernhet, H.; Gahide, G.; Klouche, K.; Bargnoux, A.-S.; Delcourt, C.; Canaud, B.; Cristol, J.-P. A cut-off value of plasma osteoprotegerin level may predict the presence of coronary artery calcifications in chronic kidney disease patients. Nephrol. Dial. Transplant. 2009, 24, 3389-3397. [CrossRef]

89. Munguía, P.; Caramelo, R.; Rubio, M.; Sahdalá, L.; Arnaudas, L.; Paul, J.; Blasco, A.; Lou, L.; Aladren, M.; Sanjuan, A.; et al. Pre-Transplant Assessment of Vascular Calcification as a Risk Factor of Mortality, Graft Loss, and Cardiovascular Events in Renal Transplant Recipients. Transplant. Proc. 2015, 47, 2368-2370. [CrossRef]

90. Nitta, K.; Hanafusa, N.; Okazaki, M.; Komatsu, M.; Kawaguchi, H.; Tsuchiya, K. Association Between Risk Factors Including Bone-Derived Biomarkers and Aortic Arch Calcification in Maintenance Hemodialysis Patients. Kidney Blood Press. Res. 2018, 43, 1554-1562. [CrossRef] [PubMed]

91. Niu, Q.; Zhao, H.; Wu, B.; Tsai, S.; Wu, J.; Zhang, M.; Lu, L.; Qiao, J.; Men, C.; Zuo, L.; et al. Abdominal aortic calcification is superior to other arteries calcification in predicting the mortality in peritoneal dialysis patients-A 8 years cohort study. BMC Nephrol. 2019, 20, 1-13. [CrossRef] [PubMed]

92. Niu, Q.; Yang, S.; Gan, L.; Zhao, H.; Zuo, L. Different type and dosage of heparin were not associated with the progression of coronary artery calcification in haemodialysis patients. Nephrol. 2020, 25, 551-558. [CrossRef] 
93. Okamoto, T.; Hatakeyama, S.; Kodama, H.; Horiguchi, H.; Kubota, Y.; Kido, K.; Momota, M.; Hosogoe, S.; Tanaka, Y.; Takashima, T.; et al. The relationship between poor nutritional status and progression of aortic calcification in patients on maintenance hemodialysis. BMC Nephrol. 2018, 19, 71. [CrossRef] [PubMed]

94. Petrovic, M.; Baralic, M.; Brkovic, V.; Arsenovic, A.; Stojanov, V.; Lalic, N.; Stanisavljevic, D.; Jankovic, A.; Radivojevic, N.; Pejanovic, S.; et al. Significance of acPWV for Survival of Hemodialysis Patients. Medicina 2020, 56, 435. [CrossRef] [PubMed]

95. Qureshi, A.R.; Olauson, H.; Witasp, A.; Haarhaus, M.L.; Brandenburg, V.; Wernerson, A.; Lindholm, B.; Söderberg, M.; Wennberg, L.; Nordfors, L.; et al. Increased circulating sclerostin levels in end-stage renal disease predict biopsy-verified vascular medial calcification and coronary artery calcification. Kidney Int. 2015, 88, 1356-1364. [CrossRef] [PubMed]

96. Raggi, P.; Bellasi, A.; Gamboa, C.; Ferramosca, E.; Ratti, C.; Block, G.A.; Muntner, P. All-cause Mortality in Hemodialysis Patients with Heart Valve Calcification. Clin. J. Am. Soc. Nephrol. 2011, 6, 1990-1995. [CrossRef]

97. Renaud, H.; Atik, A.; Hervé, M.; Morinière, P.; Hocine, C.; Belbrik, S.; Fournier, A. Evaluation of Vascular Calcinosis Risk Factors in Patients on Chronic Hemodialysis: Lack of Influence of Calcium Carbonate. Nephron 1988, 48, 28-32. [CrossRef] [PubMed]

98. Ribeiro, S.; Ramos, A.; Brandão, A.; Rebelo, J.R.; Guerra, A.; Resina, C.; Vila-Lobos, A.; Carvalho, F.; Remédio, F.; Ribeiro, F. Cardiac valve calcification in haemodialysis patients: Role of calcium-phosphate metabolism. Nephrol. Dial. Transplant. 1998, 13, 2037-2040. [CrossRef] [PubMed]

99. Roca-Tey, R.; Páez, R.; Rivas, A.; Samon, R.; Ibrik, O.; Giménez, I.; Viladoms, J. Prevalence and functional effect of arteriovenous fistula calcifications, evaluated by spiral CT in chronic haemodialysis patients. Nefrología 2009, 29, 214-221. [CrossRef]

100. Schlieper, G.; Krüger, T.; Djuric, Z.; Damjanovic, T.; Markovic, N.; Schurgers, L.; Brandenburg, V.M.; Westenfeld, R.; Dimkovic, S.; Ketteler, M.; et al. Vascular access calcification predicts mortality in hemodialysis patients. Kidney Int. 2008, 74, 1582-1587. [CrossRef] [PubMed]

101. Shu, K.-H.; Tsai, I.-C.; Ho, H.-C.; Wu, M.-J.; Chen, C.-H.; Yu, T.-M.; Chuang, Y.-W.; Huang, S.-T. Coronary Artery Calcification in Kidney Transplant Recipients with Long-term Follow-up. Transplant. Proc. 2012, 44, 687-690. [CrossRef]

102. Sigrist, M.K.; Taal, M.W.; Bungay, P.; McIntyre, C.W. Progressive Vascular Calcification over 2 Years Is Associated with Arterial Stiffening and Increased Mortality in Patients with Stages 4 and 5 Chronic Kidney Disease. Clin. J. Am. Soc. Nephrol. 2007, 2, 1241-1248. [CrossRef]

103. Stróżecki, P.; Odrowąż-Sypniewska, G.; Manitius, J. Cardiac Valve Calcifications and Left Ventricular Hypertrophy in Hemodialysis Patients. Renal Fail. 2005, 27, 733-738. [CrossRef]

104. Tangvoraphonkchai, K.; Davenport, A. Reduction in Aortic Pulse Wave Velocity Is Associated with a Short-Term Reduction in Dual-Energy X-Ray Absorptiometry Lumbar Spine Bone Mineral Density T Score. Blood Purif. 2019, 48, 346-350. [CrossRef] [PubMed]

105. Tomiyama, C.; Carvalho, A.B.; Higa, A.; Jorgetti, V.; Draibe, S.A.; Canziani, M.E.F. Coronary calcification is associated with lower bone formation rate in CKD patients not yet in dialysis treatment. J. Bone Miner. Res. 2010, 25, 499-504. [CrossRef] [PubMed]

106. Turan, M.N.; Kircelli, F.; Yaprak, M.; Sisman, A.R.; Gungor, O.; Bayraktaroğlu, S.; Ozkahya, M.; Asci, G.; Floege, J.; Ok, E. FGF-23 levels are associated with vascular calcification, but not with atherosclerosis, in hemodialysis patients. Int. Urol. Nephrol. 2016, 48, 609-617. [CrossRef] [PubMed]

107. Wang, A.Y.-M.; Wong, C.-K.; Yau, Y.-Y.; Wong, S.; Chan, I.H.-S.; Lam, C.W.-K. Skin Autofluorescence Associates with Vascular Calcification in Chronic Kidney Disease. Arter. Thromb. Vasc. Biol. 2014, 34, 1784-1790. [CrossRef] [PubMed]

108. Wang, A.Y.-M.; Wang, M.; Woo, J.; Lam, C.W.-K.; Li, P.K.-T.; Lui, S.-F.; Sanderson, J.E. Cardiac Valve Calcification as an Important Predictor for All-Cause Mortality and Cardiovascular Mortality in Long-Term Peritoneal Dialysis Patients: A Prospective Study. J. Am. Soc. Nephrol. 2003, 14, 159-168. [CrossRef]

109. Wu, C.-F.; Lee, Y.-F.; Lee, W.-J.; Su, C.-T.; Lee, L.J.-H.; Wu, K.-D.; Chen, P.-C.; Kao, T.-W. Severe aortic arch calcification predicts mortality in patients undergoing peritoneal dialysis. J. Formos. Med. Assoc. 2017, 116, 366-372. [CrossRef]

110. Yoshikawa, K.; Abe, H.; Tominaga, T.; Nakamura, M.; Kishi, S.; Matsuura, M.; Nagai, K.; Tsuchida, K.; Minakuchi, J.; Doi, T. Polymorphism in the human matrix Gla protein gene is associated with the progression of vascular calcification in maintenance hemodialysis patients. Clin. Exp. Nephrol. 2013, 17, 882-889. [CrossRef] [PubMed]

111. Zhou, Y.; Hellberg, M.; Kouidi, E.; Deligiannis, A.; Höglund, P.; Clyne, N. Relationships between abdominal aortic calcification, glomerular filtration rate, and cardiovascular risk factors in patients with non-dialysis dependent chronic kidney disease. Clin. Nephrol. 2018, 90, 380-389. [CrossRef] [PubMed]

112. Abd alamir, M.; Radulescu, V.; Goyfman, M.; Mohler, E.R., III; Gao, Y.L.; Budoff, M.J. Prevalence and correlates of mitral annular calcification in adults with chronic kidney disease: Results from CRIC study. Atherosclerosis 2015, 242, 117-122. [CrossRef]

113. Adragao, T.; Pires, A.; Lucas, C.; Birne, R.; Magalhaes, L.; Gonçalves, M.; Negrao, A.P. A simple vascular calcification score predicts cardiovascular risk in haemodialysis patients. Nephrol. Dial. Transplant. 2004, 19, 1480-1488. [CrossRef]

114. Bundy, J.D.; Cai, X.; Scialla, J.; Dobre, M.A.; Chen, J.; Hsu, C.-Y.; Leonard, M.B.; Go, A.S.; Rao, P.S.; Lash, J.P.; et al. Serum Calcification Propensity and Coronary Artery Calcification Among Patients with CKD: The CRIC (Chronic Renal Insufficiency Cohort) Study. Am. J. Kidney Dis. 2019, 73, 806-814. [CrossRef] [PubMed]

115. Chae, S.Y.; Chung, W.; Kim, Y.H.; Oh, Y.K.; Lee, J.; Choi, K.H.; Ahn, C.; Kim, Y.-S. The Correlation of Serum Osteoprotegerin with Non-Traditional Cardiovascular Risk Factors and Arterial Stiffness in Patients with Pre-Dialysis Chronic Kidney Disease: Results from the KNOW-CKD Study. J. Korean Med. Sci. 2018, 33, e322. [CrossRef] [PubMed] 
116. Dai, L.; Debowska, M.; Lukaszuk, T.; Bobrowski, L.; Barany, P.; Söderberg, M.; Thiagarajan, D.; Frostegård, J.; Wennberg, L.; Lindholm, B.; et al. Phenotypic features of vascular calcification in chronic kidney disease. J. Intern. Med. 2020, 287, 422-434. [CrossRef]

117. Floege, J.; Raggi, P.; Block, G.A.; Torres, P.U.; Csiky, B.; Naso, A.; Nossuli, K.; Moustafa, M.; Goodman, W.G.; Lopez, N.; et al. Study design and subject baseline characteristics in the ADVANCE Study: Effects of cinacalcet on vascular calcification in haemodialysis patients. Nephrol. Dial. Transplant. 2010, 25, 1916-1923. [CrossRef] [PubMed]

118. Gelev, S.; Spasovski, G.; Trajkovski, Z.; Damjanovski, G.; Amitov, V.; Selim, G.; Dzekova, P.; Sikole, A. Factors associated with various arterial calcifications in haemodialysis patients. Prilozi 2008, 29, 185-199.

119. Kestenbaum, B.R.; Adeney, K.L.; de Boer, I.H.; Ix, J.H.; Shlipak, M.G.; Siscovick, D.S. Incidence and progression of coronary calcification in chronic kidney disease: The Multi-Ethnic Study of Atherosclerosis. Kidney Int. 2009, 76, 991-998. [CrossRef] [PubMed]

120. Maia, P.R.L.; Medeiros, A.M.C.; Pereira, H.S.; Lima, K.C.; Oliveira, P.T. Presence and associated factors of carotid artery calcification detected by digital panoramic radiography in patients with chronic kidney disease undergoing hemodialysis. Oral Surg. Oral Med. Oral Pathol. Oral Radiol. 2018, 126, 198-204. [CrossRef]

121. Maréchal, C.; Coche, E.; Goffin, E.; Dragean, A.; Schlieper, G.; Nguyen, P.; Floege, J.; Kanaan, N.; Devuyst, O.; Jadoul, M. Progression of Coronary Artery Calcification and Thoracic Aorta Calcification in Kidney Transplant Recipients. Am. J. Kidney Dis. 2012, 59, 258-269. [CrossRef]

122. Moldovan, D.; Moldovan, I.; Rusu, C.; Racasan, S.; Patiu, I.M.; Brumboiu, A.; Bondor, C.I.; Parvu, L.; Kacso, I.; Orasan, R.; et al. Vascular calcifications and renal osteodystrophy in chronic hemodialysis patients: What is the relationship between them? Int. Urol. Nephrol. 2011, 43, 1179-1186. [CrossRef] [PubMed]

123. Muntner, P.; Ferramosca, E.; Bellasi, A.; Block, G.A.; Raggi, P. Development of a cardiovascular calcification index using simple imaging tools in haemodialysis patients. Nephrol. Dial. Transplant. 2007, 22, 508-514. [CrossRef]

124. Porter, C.J.; Stavroulopoulos, A.; Roe, S.D.; Pointon, K.; Cassidy, M.J. Detection of coronary and peripheral artery calcification in patients with chronic kidney disease stages 3 and 4, with and without diabetes. Nephrol. Dial. Transplant. 2007, 22, 3208-3213. [CrossRef]

125. Wang, Y.; Miao, Y.; Gong, K.; Cheng, X.; Chen, Y.; Zhao, M.-H. Plasma Complement Protein C3a Level Was Associated with Abdominal Aortic Calcification in Patients on Hemodialysis. J. Cardiovasc. Transl. Res. 2019, 12, 496-505. [CrossRef] [PubMed]

126. Wang, M.; Li, H.; You, L.; Yu, X.; Zhang, M.; Zhu, R.; Hao, C.; Zhang, Z.; Chen, J. Association of Serum Phosphorus Variability with Coronary Artery Calcification among Hemodialysis Patients. PLoS ONE 2014, 9, e93360. [CrossRef]

127. Bellasi, A.; Veledar, E.; Ferramosca, E.; Ratti, C.; Block, G.; Raggi, P. Markers of vascular disease do not differ in black and white hemodialysis patients despite a different risk profile. Atheroscler. 2008, 197, 242-249. [CrossRef] [PubMed]

128. Budoff, M.J.; Rader, D.J.; Reilly, M.; Mohler, E.R.; Lash, J.; Yang, W.; Rosen, L.; Glenn, M.; Teal, V.; Feldman, H.I. Relationship of Estimated GFR and Coronary Artery Calcification in the CRIC (Chronic Renal Insufficiency Cohort) Study. Am. J. Kidney Dis. 2011, 58, 519-526. [CrossRef]

129. Cai, H.; Lu, R.; Zhang, M.; Pang, H.; Zhu, M.; Zhang, W.; Ni, Z.; Qian, J.; Yan, Y. Serum Soluble Klotho Level Is Associated with Abdominal Aortic Calcification in Patients on Maintenance Hemodialysis. Blood Purif. 2015, 40, 120-126. [CrossRef] [PubMed]

130. Evenepoel, P.; Goffin, E.; Meijers, B.; Kanaan, N.; Bammens, B.; Coche, E.; Claes, K.; Jadoul, M. Sclerostin Serum Levels and Vascular Calcification Progression in Prevalent Renal Transplant Recipients. J. Clin. Endocrinol. Metab. 2015, 100, 4669-4676. [CrossRef]

131. Fayed, A.; Elnokeety, M.M.; Attia, K.; Din, U.A.S.E. Calcification of abdominal aorta in patients recently starting hemodialysis: A single-center experience from Egypt. Saudi J. Kidney Dis. Transplant. 2019, 30, 819-824. [CrossRef]

132. Filgueira, A.; Carvalho, A.B.; Tomiyama, C.; Higa, A.; Rochitte, C.E.; Santos, R.D.; Canziani, M.E.F. Is Coronary Artery Calcification Associated with Vertebral Bone Density in Nondialyzed Chronic Kidney Disease Patients? Clin. J. Am. Soc. Nephrol. 2011, 6, 1456-1462. [CrossRef]

133. Fusaro, M.; Gallieni, M.; Rebora, P.; Rizzo, M.A.; Luise, M.C.; Riva, H.; Bertoli, S.; Conte, F.; Stella, A.; Ondei, P.; et al. Atrial fibrillation and low vitamin D levels are associated with severe vascular calcifications in hemodialysis patients. J. Nephrol. 2016, 29, 419-426. [CrossRef]

134. Golembiewska, E.; Qureshi, A.R.; Dai, L.; Lindholm, B.; Heimbürger, O.; Söderberg, M.; Brismar, T.B.; Ripsweden, J.; Barany, P.; Johnson, R.J.; et al. Copeptin is independently associated with vascular calcification in chronic kidney disease stage 5 . $B M C$ Nephrol. 2020, 21, 1-8. [CrossRef]

135. Gruppen, M.P.; Groothoff, J.W.; Prins, M.; van der Wouw, P.; Offringa, M.; Bos, W.J.W.; Davin, J.C.; Heymans, H.S. Cardiac disease in young adult patients with end-stage renal disease since childhood: A Dutch cohort study. Kidney Int. 2003, 63, 1058-1065. [CrossRef]

136. Ho, T.-Y.; Chen, N.-C.; Hsu, C.-Y.; Huang, C.-W.; Lee, P.-T.; Chou, K.-J.; Fang, H.-C.; Chen, C.-L. Evaluation of the association of Wnt signaling with coronary artery calcification in patients on dialysis with severe secondary hyperparathyroidism. $B M C$ Nephrol. 2019, 20, 1-9. [CrossRef] [PubMed]

137. Ishimura, E.; Okuno, S.; Kitatani, K.; Maekawa, K.; Izumotani, T.; Yamakawa, T.; Jono, S.; Shoji, T.; Shioi, A.; Inaba, M.; et al. C-reactive protein is a significant predictor of vascular calcification of both aorta and hand arteries. Semin. Nephrol. 2004, 24, 408-412. [CrossRef] [PubMed] 
138. Ishimura, E.; Okuno, S.; Kitatani, K.; Kim, M.; Shoji, T.; Nakatani, T.; Inaba, M.; Nishizawa, Y. Different risk factors for peripheral vascular calcification between diabetic and non-diabetic haemodialysis patients-Importance of glycaemic control. Diabetologia 2002, 45, 1446-1448. [PubMed]

139. Manghat, P.; Souleimanova, I.; Cheung, J.; Wierzbicki, A.; Harrington, D.; Shearer, M.; Chowiencki, P.; Fogelman, I.; Nerlander, M.; Goldsmith, D.; et al. Association of bone turnover markers and arterial stiffness in pre-dialysis chronic kidney disease (CKD). Bone 2011, 48, 1127-1132. [CrossRef]

140. Wada, J.; Nakayama, K.; Nakao, K.; Takatori, Y.; Inoue, J.; Kojo, S.; Akagi, S.; Fukushima, M.; Makino, H. Long-term effect of cinacalcet hydrochloride on abdominal aortic calcification in patients on hemodialysis with secondary hyperparathyroidism. Int. J. Nephrol. Renov. Dis. 2013, 7, 25-33. [CrossRef] [PubMed]

141. Nishizawa, Y.; Mizuiri, S.; Yorioka, N.; Hamada, C.; Tomino, Y. Determinants of coronary artery calcification in maintenance hemodialysis patients. J. Artif. Organs 2015, 18, 251-256. [CrossRef]

142. Nishizawa, Y.; Jono, S.; Ishimura, E.; Shioi, A. Hyperphosphatemia and vascular calcification in end-stage renal disease. J. Ren. Nutr. 2005, 15, 178-182. [CrossRef]

143. Oprisiu, R.; Bunea, D.; Tarek, S.; Hedi, B.; Fournier, A. Progression of vascular calcification and dyslipidemia in patients on chronic hemodialysis. Am. J. Kidney Dis. 2002, 39, 209. [CrossRef] [PubMed]

144. Pateinakis, P.; Papagianni, A.; Douma, S.; Efstratiadis, G.; Memmos, D. Associations of fetuin-A and osteoprotegerin with arterial stiffness and early atherosclerosis in chronic hemodialysis patients. BMC Nephrol. 2013, 14, 122. [CrossRef]

145. Raggi, P.; Boulay, A.; Chasan-Taber, S.; Amin, N.; Dillon, M.; Burke, S.K.; Chertow, G.M. Cardiac calcification in adult hemodialysis patients: A link between end-stage renal disease and cardiovascular disease? J. Am. Coll. Cardiol. 2002, 39, 695-701. [CrossRef]

146. Schlieper, G.; Brandenburg, V.; Djuric, Z.; Damjanovic, T.; Markovic, N.; Schurgers, L.; Krüger, T.; Westenfeld, R.; Ackermann, D.; Haselhuhn, A.; et al. Risk Factors for Cardiovascular Calcifications in Non-Diabetic Caucasian Haemodialysis Patients. Kidney Blood Press. Res. 2009, 32, 161-168. [CrossRef]

147. Sharma, R.; Pellerin, D.; Gaze, D.C.; Mehta, R.L.; Gregson, H.; Streather, C.P.; Collinson, P.O.; Brecker, S.J. Mitral annular calcification predicts mortality and coronary artery disease in end stage renal disease. Atherosclerosis 2007, 191, 348-354. [CrossRef] [PubMed]

148. Sigrist, M.; Bungay, P.; Taal, M.; McIntyre, C.W. Vascular calcification and cardiovascular function in chronic kidney disease. Nephrol. Dial. Transplant. 2006, 21, 707-714. [CrossRef]

149. Stavroulopoulos, A.; Porter, C.J.; Pointon, K.; Monaghan, J.M.; Roe, S.D.; Cassidy, M.J. Evolution of coronary artery calcification in patients with chronic kidney disease Stages 3 and 4, with and without diabetes. Nephrol. Dial. Transplant. 2011, 26, 2582-2589. [CrossRef] [PubMed]

150. Sumida, Y.; Nakayama, M.; Nagata, M.; Nakashita, S.; Suehiro, T.; Kaizu, Y.; Ikeda, H.; Izumaru, K. Carotid artery calcification and atherosclerosis at the initiation of hemodialysis in patients with end-stage renal disease. Clin. Nephrol. 2010, 73, 360-369. [CrossRef]

151. Tamei, N.; Ogawa, T.; Ishida, H.; Ando, Y.; Nitta, K. Serum fibroblast growth factor-23 levels and progression of aortic arch calcification in non-diabetic patients on chronic hemodialysis. J. Atheroscler. Thromb. 2011, 18, 217-223. [CrossRef]

152. Turan, M.N.; Gungor, O.; Asci, G.; Kircelli, F.; Acar, T.; Yaprak, M.; Ceylan, N.; Demirci, M.S.; Bayraktaroglu, S.; Töz, H.; et al. Epicardial adipose tissue volume and cardiovascular disease in hemodialysis patients. Atherosclerosis 2013, 226, 129-133. [CrossRef] [PubMed]

153. Vipattawat, K.; Kitiyakara, C.; Phakdeekitcharoen, B.; Kantachuvesiri, S.; Sumethkul, V.; Jirasiritham, S.; Stitchantrakul, W.; Disthabanchong, S. Vascular calcification in long-term kidney transplantation. Nephrology 2014, 19, 251-256. [CrossRef] [PubMed]

154. Yamada, S.; Inaba, M.; Shidara, K.; Okada, S.; Emoto, M.; Ishimura, E.; Nishizawa, Y. Association of glycated albumin, but not glycated hemoglobin, with peripheral vascular calcification in hemodialysis patients with type 2 diabetes. Life Sci. 2008, 83, 516-519. [CrossRef] [PubMed]

155. Jung, H.H.; Kim, S.-W.; Han, H. Inflammation, mineral metabolism and progressive coronary artery calcification in patients on haemodialysis. Nephrol. Dial. Transplant. 2006, 21, 1915-1920. [CrossRef]

156. Aoun, M.; Makki, M.; Azar, H.; Matta, H.; Chelala, D.N. High Dephosphorylated-Uncarboxylated MGP in Hemodialysis patients: Risk factors and response to vitamin K2, A pre-post intervention clinical trial. BMC Nephrol. 2017, 18, 1-10. [CrossRef]

157. Axelsson, J.; Wang, X.; Ketteler, M.; Qureshi, A.R.; Heimbürger, O.; Bárány, P.; Lindholm, B.; Nordfors, L.; Stenvinkel, P. Is Fetuin-A/ $\alpha 2$-Heremans-Schmid Glycoprotein Associated with the Metabolic Syndrome in Patients with Chronic Kidney Disease? Am. J. Nephrol. 2008, 28, 669-676. [CrossRef] [PubMed]

158. Block, G.A.; Hulbert-Shearon, E.T.; Levin, N.W.; Port, F.K. Association of serum phosphorus and calcium x phosphate product with mortality risk in chronic hemodialysis patients: A national study. Am. J. Kidney Dis. 1998, 31, 607-617. [CrossRef] [PubMed]

159. Buiten, M.S.; De Bie, M.K.; Krijger, A.B.-D.; Van Dam, B.; Dekker, F.; Jukema, J.W.; Rabelink, T.J.; Rotmans, J.I. Soluble Klotho is not independently associated with cardiovascular disease in a population of dialysis patients. BMC Nephrol. 2014, 15, 197. [CrossRef]

160. Chao, C.; Yuan, T.; Yeh, H.; Chen, H.; Huang, J.; Chen, H. Risk Factors Associated with Altered Circulating MicroRNA-125b and Their Influences on Uremic Vascular Calcification Among Patients with End-Stage Renal Disease. J. Am. Hear. Assoc. 2019, 8 , e010805. [CrossRef] 
161. Chen, H.-Y.; Chiu, Y.-L.; Hsu, S.-P.; Pai, M.-F.; Yang, J.-Y.; Peng, Y.-S. Low serum fetuin A levels and incident stroke in patients with maintenance haemodialysis. Eur. J. Clin. Investig. 2013, 43, 387-396. [CrossRef] [PubMed]

162. Claes, K.J.; Viaene, L.; Heye, S.; Meijers, B.; D’Haese, P.; Evenepoel, P. Sclerostin: Another Vascular Calcification Inhibitor? J. Clin. Endocrinol. Metab. 2013, 98, 3221-3228. [CrossRef] [PubMed]

163. Gonçalves, F.L.C.; Elias, R.M.; Dos Reis, L.M.; Graciolli, F.G.; Zampieri, F.G.; Oliveira, R.B.; Jorgetti, V.; Moysés, R.M.A. Serum sclerostin is an independent predictor of mortality in hemodialysis patients. BMC Nephrol. 2014, 15, 1-7. [CrossRef]

164. González-Parra, E.; Álvaro, A.; Lorenzo, O.; Tarín, N.; González-Casaus, M.L.; Cristóbal, C.; Huelmos, A.; Fernandez, J.L.T.; Pello, A.M.; Carda, R.; et al. Important abnormalities of bone mineral metabolism are present in patients with coronary artery disease with a mild decrease of the estimated glomerular filtration rate. J. Bone Miner. Metab. 2016, 34, 587-598. [CrossRef] [PubMed]

165. Gupta, V.; Ekundayo, O.; Nemeth, Z.K.; Yang, Y.; Covic, A.; Mathe, Z.; Kovesdy, C.P.; Molnar, M.Z.; Mucsi, I. Association between serum osteoprotegerin level and mortality in kidney transplant recipients-A prospective observational cohort study. Transpl. Int. 2021, 34, 844-854. [CrossRef]

166. Holden, R.M.; Booth, S.L.; Tuttle, A.; James, P.D.; Morton, A.R.; Hopman, W.M.; Nolan, R.; Garland, J.S. Sequence Variation in Vitamin K Epoxide Reductase Gene Is Associated with Survival and Progressive Coronary Calcification in Chronic Kidney Disease. Arter. Thromb. Vasc. Biol. 2014, 34, 1591-1596. [CrossRef]

167. Ikee, R.; Toyoyama, T.; Endo, T.; Tsunoda, M.; Hashimoto, N. Impact of sevelamer hydrochloride on serum magnesium concentrations in hemodialysis patients. Magnes Res. 2016, 29, 184-190. [CrossRef]

168. Jean, G.; Charra, B.; Chazot, C. Vitamin D Deficiency and Associated Factors in Hemodialysis Patients. J. Ren. Nutr. 2008, 18, 395-399. [CrossRef]

169. Jean, G.; Lataillade, D.; Genet, L.; Legrand, E.; Kuentz, F.; Moreau-Gaudry, X.; Fouque, D. Association between Very Low PTH Levels and Poor Survival Rates in Haemodialysis Patients: Results from the French ARNOS Cohort. Nephron Clin. Pract. 2011, 118, c211-c216. [CrossRef]

170. Karsli Ceppioğlu, S.; Yurdun, T.; Canbakan, M. Assessment of Matrix Gla Protein, Klotho Gene Polymorphisms, and Oxidative Stress in Chronic Kidney Disease. Renal Fail. 2011, 33, 866-874. [CrossRef]

171. Kato, A.; Odamaki, M.; Ishida, J.; Hishida, A. Relationship between Serum Pre-B Cell Colony-Enhancing Factor/Visfatin and Atherosclerotic Parameters in Chronic Hemodialysis Patients. Am. J. Nephrol. 2009, 29, 31-35. [CrossRef]

172. Kuo, T.-H.; Lin, W.-H.; Chao, J.-Y.; Wu, A.-B.; Tseng, C.-C.; Chang, Y.-T.; Liou, H.-H.; Wang, M.-C. Serum sclerostin levels are positively related to bone mineral density in peritoneal dialysis patients: A cross-sectional study. BMC Nephrol. 2019, 20, 1-10. [CrossRef]

173. Liabeuf, S.; Glorieux, G.; Lenglet, A.; Diouf, M.; Schepers, E.; Desjardins, L.; Choukroun, G.; Vanholder, R.; Massy, Z.A.; on behalf of the European Uremic Toxin (EUTox) Work Group. Does P-Cresylglucuronide Have the Same Impact on Mortality as Other Protein-Bound Uremic Toxins? PLoS ONE 2013, 8, e67168. [CrossRef]

174. Metry, G.; Stenvinkel, P.; Qureshi, A.R.T.; Carrero, J.J.; Yilmaz, M.I.; Bárány, P.; Snaedal, S.; Heimbürger, O.; Lindholm, B.; Suliman, M.E. Low serum fetuin-A concentration predicts poor outcome only in the presence of inflammation in prevalent haemodialysis patients. Eur. J. Clin. Investig. 2008, 38, 804-811. [CrossRef] [PubMed]

175. Nakashima, A.; Carrero, J.J.; Qureshi, A.R.T.; Hirai, T.; Takasugi, N.; Ueno, T.; Taniguchi, Y.; Lindholm, B.; Yorioka, N. Plasma osteoprotegerin, arterial stiffness, and mortality in normoalbuminemic Japanese hemodialysis patients. Osteoporos. Int. 2011, 22, 1695-1701. [CrossRef]

176. Nemeth, Z.K.; Mardare, N.G.; Czira, M.E.; Deak, G.; Kiss, I.; Mathe, Z.; Remport, A.; Ujszaszi, A.; Covic, A.; Molnar, M.Z.; et al. Serum osteoprotegerin is associated with pulse pressure in kidney transplant recipients. Sci. Rep. 2015, 5, 14518. [CrossRef] [PubMed]

177. Nishiura, R.; Fujimoto, S.; Sato, Y.; Yamada, K.; Hisanaga, S.; Hara, S.; Nakao, H.; Kitamura, K. Elevated Osteoprotegerin Levels Predict Cardiovascular Events in New Hemodialysis Patients. Am. J. Nephrol. 2009, 29, 257-263. [CrossRef] [PubMed]

178. Okamoto, T.; Tsutaya, C.; Hatakeyama, S.; Konishi, S.; Okita, K.; Tanaka, Y.; Imanishi, K.; Takashima, T.; Saitoh, F.; Suzuki, T.; et al. Low serum butyrylcholinesterase is independently related to low fetuin-A in patients on hemodialysis: A cross-sectional study. Int. Urol. Nephrol. 2018, 50, 1713-1720. [CrossRef]

179. Park, S.; Lee, C.J.; Jhee, J.H.; Yun, H.; Kim, H.; Jung, S.; Kee, Y.K.; Yoon, C.; Park, J.T.; Kim, H.C.; et al. Extracellular Fluid Excess Is Significantly Associated with Coronary Artery Calcification in Patients with Chronic Kidney Disease. J. Am. Hear. Assoc. 2018, 7 , e008935. [CrossRef]

180. Ramalho, J.; Petrillo, E.M.; Takeichi, A.P.M.; Moyses, R.M.A.; Titan, S.M. Calcitriol and FGF-23, but neither PTH nor sclerostin, are associated with calciuria in CKD. Int. Urol. Nephrol. 2019, 51, 1823-1829. [CrossRef]

181. Scialla, J.J.; Leonard, M.B.; Townsend, R.R.; Appel, L.; Wolf, M.; Budoff, M.J.; Chen, J.; Lustigova, E.; Gadegbeku, C.A.; Glenn, M.; et al. Correlates of Osteoprotegerin and Association with Aortic Pulse Wave Velocity in Patients with Chronic Kidney Disease. Clin. J. Am. Soc. Nephrol. 2011, 6, 2612-2619. [CrossRef] [PubMed]

182. Schlieper, G.R.; Westenfeld, R.; Krüger, T.; Cranenburg, E.C.M.; Magdeleyns, E.J.; Brandenburg, V.M.; Djuric, Z.; Damjanovic, T.; Ketteler, M.; Vermeer, C.; et al. Circulating Nonphosphorylated Carboxylated Matrix Gla Protein Predicts Survival in ESRD. J. Am. Soc. Nephrol. 2011, 22, 387-395. [CrossRef]

183. Sigrist, M.K.; Levin, A.; Er, L.; McIntyre, C.W. Elevated osteoprotegerin is associated with all-cause mortality in CKD stage 4 and 5 patients in addition to vascular calcification. Nephrol. Dial. Transplant. 2009, 24, 3157-3162. [CrossRef] 
184. Stenvinkel, P.; Wang, K.; Qureshi, A.R.; Axelsson, J.; Pecoits-Filho, R.; Gao, P.; Barany, P.; Lindholm, B.; Jogestrand, T.; Heimberger, O.; et al. Low fetuin-A levels are associated with cardiovascular death: Impact of variations in the gene encoding fetuin. Kidney Int. 2005, 67, 2383-2392. [CrossRef] [PubMed]

185. Stolic, R.V.; Jovanovic, A.; Trajkovic, G.Z.; Kostić, M.M.; Odalovic, A.M.; Sovtic, S.R.; Sipic, M.V.; Pajovic, S.D.; SojevicTimotijevic, Z.N. Is low magnesium a clue to arteriovenous fistula complications in hemodialysis? Int. Urol. Nephrol. 2016, 48, 773-779. [CrossRef]

186. Thambiah, S.C.; Roplekar, R.; Manghat, P.; Fogelman, I.; Fraser, W.D.; Goldsmith, D.; Hampson, G. Circulating Sclerostin and Dickkopf-1 (DKK1) in Predialysis Chronic Kidney Disease (CKD): Relationship with Bone Density and Arterial Stiffness. Calcif. Tissue Int. 2012, 90, 473-480. [CrossRef]

187. Ulusoy, S.; Özkan, G.; Mentese, A.; Yavuz, A.; Karahan, S.C.; Sümer, A.U. Signal peptide-CUB-EGF domain-containing protein 1 (SCUBE1) level in hemodialysis patients and parameters affecting that level. Clin. Biochem. 2012, 45, 1444-1449. [CrossRef]

188. Viaene, L.; Behets, G.; Claes, K.; Meijers, B.; Blocki, F.; Brandenburg, V.; Evenepoel, P.; D’Haese, P.C. Sclerostin: Another bone-related protein related to all-cause mortality in haemodialysis? Nephrol. Dial. Transplant. 2013, 28, 3024-3030. [CrossRef]

189. Zhang, A.-H.; Guo, W.-K.; Yu, L.; Liu, W.-H. Relationship of Serum Soluble Klotho Levels and Echocardiographic Parameters in Patients on Maintenance Hemodialysis. Kidney Blood Press. Res. 2019, 44, 396-404. [CrossRef] [PubMed]

190. Zhang, Q.; Sun, L.; Jin, L. Association Between Angiotensin-Converting Enzyme 2 and Coronary Artery Calcification in Patients on Maintenance Hemodialysis Therapy. Ther. Apher. Dial. 2015, 19, 466-470. [CrossRef]

191. Zou, Y.; Yang, M.; Wang, J.; Cui, L.; Jiang, Z.; Ding, J.; Li, M.; Zhou, H. Association of sclerostin with cardiovascular events and mortality in dialysis patients. Ren. Fail. 2020, 42, 282-288. [CrossRef] [PubMed]

192. Zou, J.; Yu, Y.; Wu, P.; Lin, F.-J.; Yao, Y.; Xie, Y.; Jiang, G.-R. Serum phosphorus is related to left ventricular remodeling independent of renal function in hospitalized patients with chronic kidney disease. Int. J. Cardiol. 2016, 221, 134-140. [CrossRef]

193. Chao, C.-T.; Lin, S.-H. Uremic Vascular Calcification: The Pathogenic Roles and Gastrointestinal Decontamination of Uremic Toxins. Toxins 2020, 12, 812. [CrossRef]

194. Nakahara, T.; Dweck, M.R.; Narula, N.; Pisapia, D.; Narula, J.; Strauss, H.W. Coronary Artery Calcification: From Mechanism to Molecular Imaging. JACC Cardiovasc. Imaging 2017, 10, 582-593. [CrossRef] [PubMed]

195. Amann, K. Media Calcification and Intima Calcification Are Distinct Entities in Chronic Kidney Disease. Clin. J. Am. Soc. Nephrol. 2008, 3, 1599-1605. [CrossRef]

196. Lee, J.J.; Pedley, A.; Hoffmann, U.; Massaro, J.M.; O’Donnell, C.J.; Benjamin, E.J.; Long, M.T. Longitudinal Associations of Pericardial and Intrathoracic Fat with Progression of Coronary Artery Calcium (from the Framingham Heart Study). Am. J. Cardiol. 2018, 121, 162-167. [CrossRef] [PubMed]

197. Sui, Y.-B.; Chang, J.-R.; Chen, W.-J.; Zhao, L.; Zhang, B.-H.; Yu, Y.-R.; Tang, C.-S.; Yin, X.-H.; Qi, Y.-F. Angiotensin-(1-7) inhibits vascular calcification in rats. Peptides 2013, 42, 25-34. [CrossRef]

198. McCabe, K.M.; Zelt, J.G.; Kaufmann, M.; Laverty, K.; Ward, E.; Barron, H.; Jones, G.; Adams, M.A.; Holden, R.M. Calcitriol Accelerates Vascular Calcification Irrespective of Vitamin K Status in a Rat Model of Chronic Kidney Disease with Hyperphosphatemia and Secondary Hyperparathyroidism. J. Pharmacol. Exp. Ther. 2018, 366, 433-445. [CrossRef]

199. Almeida, Y.E.; Fessel, M.R.; Carmo, L.S.D.; Jorgetti, V.; Farias-Silva, E.; Pescatore, L.A.; Gamarra, L.; Andrade, M.C.; SimplicioFilho, A.; Mangueira, C.L.P.; et al. Excessive cholecalciferol supplementation increases kidney dysfunction associated with intrarenal artery calcification in obese insulin-resistant mice. Sci. Rep. 2020, 10, 87. [CrossRef]

200. De Maré, A.; Maudsley, S.; Azmi, A.; Hendrickx, J.O.; Opdebeeck, B.; Neven, E.; D’Haese, P.C.; Verhulst, A. Sclerostin as Regulatory Molecule in Vascular Media Calcification and the Bone-Vascular Axis. Toxins 2019, 11, 428. [CrossRef]

201. Roumeliotis, S.; Dounousi, E.; Salmas, M.; Eleftheriadis, T.; Liakopoulos, V. Vascular Calcification in Chronic Kidney Disease: The Role of Vitamin K- Dependent Matrix Gla Protein. Front. Med. (Lausanne) 2020, 7, 154. [CrossRef] [PubMed]

202. Hu, M.C.; Shi, M.; Zhang, J.; Quiñones, H.; Griffith, C.; Kuro-o, M.; Moe, O.W. Klotho deficiency causes vascular calcification in chronic kidney disease. J. Am. Soc. Nephrol. 2011, 22, 124-136. [CrossRef] [PubMed]

203. Ceponiene, I.; Li, D.; El Khoudary, S.R.; Nakanishi, R.; Stein, J.H.; Wong, N.D.; Nezarat, N.; Kanisawa, M.; Rahmani, S.; Osawa, K.; et al. Association of Coronary Calcium, Carotid Wall Thickness, and Carotid Plaque Progression with Low-Density Lipoprotein and High-Density Lipoprotein Particle Concentration Measured by Ion Mobility (From Multiethnic Study of Atherosclerosis [MESA]). Am. J. Cardiol. 2021, 142, 52-58. [CrossRef]

204. Parhami, F.; Basseri, B.; Hwang, J.; Tintut, Y.; Demer, L.L. High-density lipoprotein regulates calcification of vascular cells. Circ. Res. 2002, 91, 570-576. [CrossRef] [PubMed]

205. Osako, M.K.; Nakagami, H.; Koibuchi, N.; Shimizu, H.; Nakagami, F.; Koriyama, H.; Shimamura, M.; Miyake, T.; Rakugi, H.; Morishita, R. Estrogen inhibits vascular calcification via vascular RANKL system: Common mechanism of osteoporosis and vascular calcification. Circ. Res. 2010, 107, 466-475. [CrossRef] [PubMed]

206. Balica, M.; Boström, K.; Shin, V.; Tillisch, K.; Demer, L.L. Calcifying subpopulation of bovine aortic smooth muscle cells is responsive to 17 beta-estradiol. Circulation. 1997, 95, 1954-1960. [CrossRef]

207. Woodward, H.J.; Zhu, D.; Hadoke, P.W.F.; MacRae, V.E. Regulatory Role of Sex Hormones in Cardiovascular Calcification. Int. J. Mol. Sci. 2021, 22, 4620. [CrossRef] [PubMed] 
208. Ray, M.; Jovanovich, A. Mineral Bone Abnormalities and Vascular Calcifications. Adv. Chronic Kidney Dis. 2019, 26, 409-416. [CrossRef]

209. Jia, J.; Zhou, H.; Zeng, X.; Feng, S. Estrogen stimulates osteoprotegerin expression via the suppression of miR-145 expression in MG-63 cells. Mol. Med. Rep. 2017, 15, 1539-1546. [CrossRef] 